\title{
A unified framework for robust modelling of financial markets in discrete time
}

\author{
Jan Obłój ${ }^{1}$ Johannes Wiesel ${ }^{2}$
}

Received: 17 March 2020 / Accepted: 16 December 2020 / Published online: 14 June 2021

(c) The Author(s) 2021

\begin{abstract}
We unify and establish equivalence between the pathwise and the quasi-sure approaches to robust modelling of financial markets in finite discrete time. In particular, we prove a fundamental theorem of asset pricing and a superhedging theorem which encompass the formulations of Bouchard and Nutz [12] and Burzoni et al. [13]. In bringing the two streams of literature together, we examine and compare their many different notions of arbitrage. We also clarify the relation between robust and classical $\mathbb{P}$-specific results. Furthermore, we prove when a superhedging property with respect to the set of martingale measures supported on a set $\Omega$ of paths may be extended to a pathwise superhedging on $\Omega$ without changing the superhedging price.
\end{abstract}

Keywords Robust pricing and hedging · Superhedging · Model-independent arbitrage $\cdot$ Dynamic programming principle

Mathematics Subject Classification (2010) 91G20 • 60G42

JEL Classification C61 · C65 - G12 · G13

\section{Introduction}

Mathematical models of financial markets are of great significance in economics and finance and have played a key role in the theory of pricing and hedging derivatives

We gratefully acknowledge funding received from the European Research Council under the European Union's Seventh Framework Programme (FP7/2007-2013)/ERC grant agreement no. 335421) and from St. John's College in Oxford.

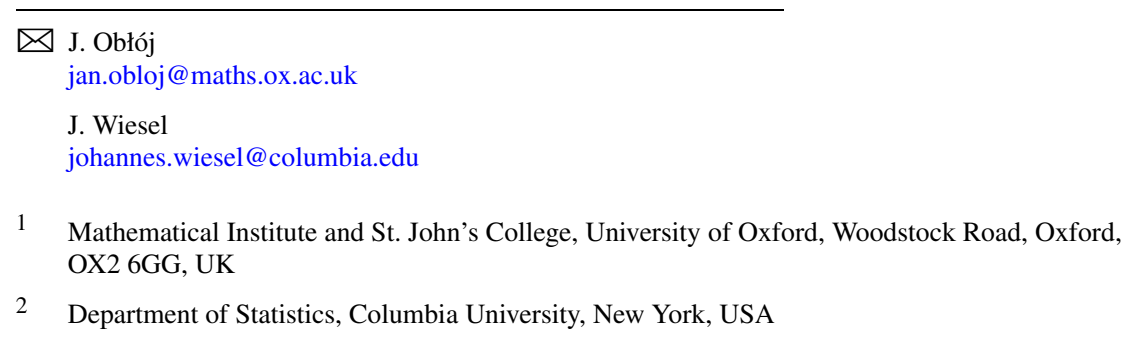


and of risk management. Classical models, going back to Samuelson [37] and Black and Scholes [9] in continuous time, specify a fixed probability measure $\mathbb{P}$ to describe the asset price dynamics. They led to a powerful theory of complete, and later incomplete, financial markets. The original models have undergone a myriad of variations, including, amongst others, local and stochastic volatility models, and have been widely applied. However, they also faced important criticism for ignoring the issue of model uncertainty, particularly so in the wake of the 2007/08 financial crisis. Consequently, inspired by the theoretical developments going back to Knight [29, Chap. VII], new modelling approaches emerged which aim to address this fundamental issue. These can be broadly divided into two streams based on the so-called quasi-sure and pathwise approaches, respectively.

The quasi-sure approach introduces a set $\mathfrak{P}$ of priors representing possible market scenarios. These priors can be very different, and $\mathfrak{P}$ typically contains measures which are mutually singular. This presents significant mathematical challenges and led to the theory of quasi-sure stochastic analysis (see e.g. Peng [33, Chap. 3], Denis and Martini [25]). In finite discrete time, this framework was abstracted in Bouchard and Nutz [12], which we call the quasi-sure formulation in the rest of this paper. By varying the set $\mathfrak{P}$ of probability measures between the "extreme" cases of one fixed probability measure, $\mathfrak{P}=\{\mathbb{P}\}$, and that of considering all probability measures, $\mathfrak{P}=\mathcal{P}(X)$, this formulation allows widely different specifications of market dynamics. The quasi-sure approach has been employed to consider model uncertainty along with market frictions and other related problems; see e.g. Bayraktar and Zhou [6], Bayraktar and Zhang [5].

The pathwise approach addresses Knightian uncertainty in market modelling by describing the set of market scenarios in the absence of a probability measure or any similar relative weighting of such scenarios. It is also referred to as the pointwise, or $\omega$-by- $\omega$, approach and bears similarity to the way central banks carry out stress tests using scenario generators. In finite discrete time, a suitable theory was obtained in Burzoni et al. [13], based on earlier developments in Burzoni et al. [15, 14]. The methodology builds on the notion of prediction sets introduced in Mykland [31] and used in continuous time in Hou and Obłój [28]. The particular case of including all scenarios is often referred to as the model-independent framework and was pioneered in Davis and Hobson [23], Cox and Obłój [21] and Acciaio et al. [1]. From here, a further model specification is carried out by including additional assumptions, which represent the different agents' beliefs. In this manner, paths deemed impossible by all agents are eliminated; see Cassese [18]. The remaining set of paths is then called the prediction set, or the model. The pathwise approach is also the starting point of the game-theoretical perspective - the hedger decides on a strategy and nature chooses an adverse scenario (the path) - as developed in Shafer and Vovk [38, Chap. 10], Vovk [41]; see also Smirnov [39].

Both approaches, the quasi-sure and the pathwise, allow thus to interpolate between the two ends of the modelling spectrum, as identified by Merton [30]: the model-independent and the model-specific settings (see Fig. 1). In doing so, they allow capturing how their outputs change in terms of adding or removing modelling assumptions, thus allowing us to quantify the impact and risk that a given set of assumptions bear on the problem at hand; see Cont [19]. Both approaches were successful in developing suitable notions of arbitrage and extending the core results from the 


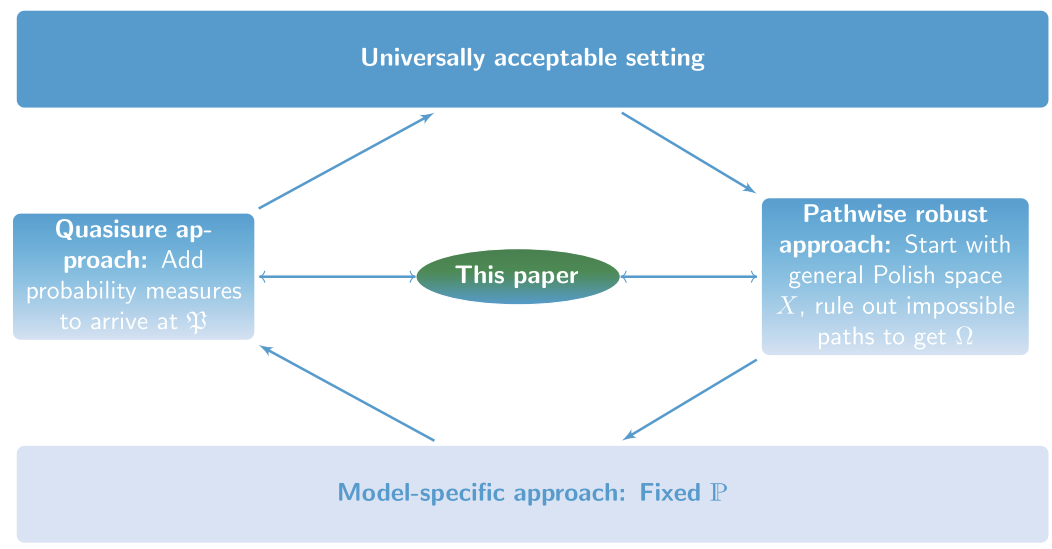

Fig. 1 Different approaches to modelling financial markets

classical $\mathbb{P}$-a.s. setting to their more general context. In particular, in both approaches, it is possible to establish a fundamental theorem of asset pricing of the form

$$
\text { no arbitrage } \Longleftrightarrow \text { existence of martingale measures } \mathbb{Q}
$$

and a superhedging (duality) theorem of the form

$\sup _{\mathbb{Q}} \mathbb{E}_{\mathbb{Q}}[g]=\inf \{x: x$ is the initial capital of a superhedging strategy for $g\}$.

Our main contribution is to unify these two approaches to model uncertainty and to build an overarching understanding of the topic. We show that under mild technical assumptions, the pathwise and quasi-sure fundamental theorems of asset pricing and superhedging dualities can be inferred from one another and are thus equivalent. Our statements follow a meta-structure outlined below.

Meta-Theorem 1.1 Suppose we are in the quasi-sure setting with a given set $\mathfrak{P}$ of priors. Then there exists a suitable selection $\Omega^{\mathfrak{P}}$ of scenarios such that the pathwise result for $\Omega^{\mathfrak{P}}$ implies the quasi-sure result for $\mathfrak{P}$.

Conversely, suppose we are given a selection $\Omega$ of scenarios. Then there is a set $\mathfrak{P}^{\Omega}$ of priors such that the quasi-sure result for $\mathfrak{P}^{\Omega}$ implies the pathwise result for $\Omega$.

We believe that establishing such equivalence statements is of prime interest for the field. They are mathematically elegant and offer a sense of closure to the different competing narratives. Most importantly, they offer significant novel insights into the core objects in both approaches. Our work also builds and clarifies the links to the classical model-specific setting. In particular, when transposing the results from the pointwise to the quasi-sure setup, the key technical analytic product structure assumption in Bouchard and Nutz [12], see Definition 2.1 below, is deduced naturally from the analyticity of the set of scenarios in Burzoni et al. [13]. When establishing the superhedging theorem, we not only show that the pathwise superhedging 
price of $g$ is equal to the quasi-sure one, but also that both are equal to the modelspecific $\mathbb{P}^{g}$-superhedging price, for some $\mathbb{P}^{g}$ which depends on the setting, i.e., on $\mathfrak{P}$ or equivalently on $\Omega$, but also on the payoff $g$. Intuitively, $\mathbb{P}^{g}$ just has to charge all the most adverse scenarios from the perspective of hedging $g$. For a continuous $g$, in the pathwise approach, one could hope that any measure with "full support" could be suitable; see Sect. 3.1 below and Carassus et al. [17, Proposition 3.7]. However, in general, determining such a measure $\mathbb{P}^{g}$ is not straightforward and relies on separability of $\mathbb{R}^{d}$ together with measurable selection results: it is constructed by identifying the portion of the $\mathfrak{P}$-quasi-sure support on which the $\mathfrak{P}$-q.s. superhedging constraint is binding. Finally, the key implication in the proof of the robust fundamental theorem of asset pricing, i.e., 5) $\Rightarrow 1$ ) in Theorem 2.7 below, is obtained by carefully constructing a suitable $\mathbb{P} \in \mathfrak{P}$ which does not admit an arbitrage in the classical sense and hence admits an equivalent martingale measure. A similar result was established in parallel and independent work by Blanchard and Carassus [10, Theorem 3.8 and Remark 3.34], where they show a different characterisation of quasisure arbitrages by their notion of WA(P) (see Sect. 3.2). In particular, they establish equivalence of a quasi-sure, a geometric and a quantitative notion of arbitrage [10, Theorem 3.24]).

Furthermore, we survey and relate the concepts of arbitrage used in both approaches. We provide an extensive list of arbitrage notions introduced and used across the literature on robust finance and establish clear relations between them. We also investigate in detail the notion of pathwise superhedging. As noted in Burzoni et al. [15], the pathwise superhedging duality does not hold for general claims $g$ when superhedging on a general set $\Omega$ is required. Instead, one has to consider hedging on a smaller "efficient" set $\Omega *$ (defined as the largest set supported by martingale measures and contained in $\Omega$ ) to retain the pricing-hedging duality. We clarify when this is necessary and when one can extend the superhedging duality from $\Omega^{*}$ to $\Omega$. Intuitively, since there are arbitrage opportunities on $\Omega \backslash \Omega^{*}$, one could try to superhedge the claim $g$ on $\Omega \backslash \Omega^{*}$ without any additional cost by implementing an arbitrage strategy. We provide a number of counterexamples to show that this idea is not feasible in general and link this to measurability constraints on arbitrage strategies, which were also encountered in Burzoni et al. [14]. We then show that the above-mentioned intuition is only true for essentially uniformly continuous $g$ under certain regularity conditions on $\Omega$.

The rest of the paper is organised as follows. Section 2 contains the main results. First, in Sect. 2.1, we introduce the general setup in which we work. Then, in Sect. 2.2, we establish our version of the robust fundamental theorem of asset pricing which unifies the quasi-sure and pathwise perspectives. In Sect. 2.3, we state a robust superhedging theorem. Section 3 presents complementary results: on extending the superhedging duality from $\Omega^{*}$ to $\Omega$ without additional cost, on different notions of arbitrage, and in particular on the relation between two strong notions of pathwise arbitrage. Finally, Sect. 4 contains technical results and most of the proofs. In particular, we give the proofs of Theorems 2.5 and 2.7 in Sect. 4.1 and of Theorem 2.9 in Sect. 4.2. 


\section{Unified framework for robust modelling of financial markets}

\subsection{Trading strategies and pricing measures}

We use notation similar to Bouchard and Nutz [12] and work in their setting; so we only recall the main objects of interest here and refer to Bouchard and Nutz [12] and Bertsekas and Shreve [8, Chap. 7] for technical details. Let $T \in \mathbb{N}$ and $X_{1}$ be a Polish space. We define for $t \in\{1, \ldots, T\}$ the Cartesian product $X_{t}:=X_{1}^{t}$ and define $X:=X_{T}$, with the convention that $X_{0}$ is a singleton. We denote by $\mathcal{B}(X)$ the Borel sets on $X$, by $\mathcal{P}(X)$ the set of probability measures on $\mathcal{B}(X)$ and define the function $\operatorname{proj}_{t}: X \rightarrow X_{1}$ which projects $\omega \in X$ to the $t$ th coordinate, i.e., $\operatorname{proj}_{t}(\omega)=\omega_{t}$.

Next we specify the financial market. Let $d \in \mathbb{N}, \mathbb{F}=\left(\mathcal{F}_{t}\right)_{t=0,1, \ldots, T}$ be an arbitrary filtration and let $S_{t}=\left(S_{t}^{1}, \ldots, S_{t}^{d}\right): X_{t} \rightarrow \mathbb{R}^{d}$ be Borel-measurable, $t=0,1, \ldots, T$, and adapted. All prices are given in units of a tradable numeraire $S^{0}$ which itself is thus normalised, $S_{t}^{0} \equiv 1, t=0,1, \ldots, T$. This is a standard setup which we adopt here, but we refer to Bálint and Schweizer [2] for a discussion of alternative market specifications. Trading strategies $\mathcal{H}(\mathbb{F})$ are defined as the set of $\mathbb{F}$-predictable $\mathbb{R}^{d}$-valued processes. All trading is frictionless and self-financing. Given $H \in \mathcal{H}(\mathbb{F})$, we denote

$$
H \cdot S_{t}=\sum_{u=1}^{t} H_{u} \Delta S_{u}
$$

which represents the cashflow at time $t$ from trading using $H$. Above and throughout, $H$ is a row vector, $S$ is a column vector and 1 denotes either a scalar or a column vector $(1, \ldots, 1)^{\top}$. We let $\Phi$ denote the vector of payoffs of $k$ statically traded assets $\Phi=\left(\phi_{\lambda}: \lambda \in\{1, \ldots, k\}\right)$. For notational convenience, we often identify $\Phi$ with the set of its elements. We assume that each $\phi \in \Phi$ is Borel-measurable. When there are no statically traded assets, we write $\Phi=0$. These assets, which we think of as options, can only be bought or sold at time zero (without loss of generality at zero cost) and are held until maturity $T$. A trading position $h \in \mathbb{R}^{k}$ in these assets generates the payoff $h \cdot \Phi=\sum_{\lambda=1}^{k} h_{\lambda} \phi_{\lambda}$ at time $T$. We call a pair $(h, H) \in \mathbb{R}^{k} \times \mathcal{H}(\mathbb{F})$ a semistatic trading strategy. The class of such strategies is denoted $\mathcal{A}_{\Phi}(\mathbb{F}):=\mathbb{R}^{k} \times \mathcal{H}(\mathbb{F})$. For technical reasons, we also introduce the level sets of $S$, which are denoted by

$$
\Sigma_{t}^{\omega}=\left\{\tilde{\omega} \in X: S_{0: t}(\tilde{\omega})=S_{0: t}(\omega)\right\}
$$

for $t \in\{0, \ldots, T\}$ and $\omega \in X_{t}$, where $S_{0: t}:=\left(S_{0}, \ldots, S_{t}\right)$. Finally, we denote by $\mathbb{F}^{0}=\left(\mathcal{F}_{t}^{0}\right)_{t=0,1, \ldots, T}$ the natural filtration generated by $S$ and let $\mathcal{F}_{t}^{\mathcal{U}}$ be the universal completion of $\mathcal{F}_{t}^{0}, t=0,1, \ldots, T$, i.e., the intersection of all possible completions of $\mathcal{F}_{t}^{0}$. Furthermore we write $\left(X, \mathcal{F}^{\mathcal{U}}\right)$ for $\left(X_{T}, \mathcal{F}_{T}^{\mathcal{U}}\right)$ and often consider $\left(X_{t}, \mathcal{F}_{t}^{\mathcal{U}}\right)$ as a subspace of $\left(X, \mathcal{F}^{\mathcal{U}}\right)$.

Within this setup, the literature on robust pricing and hedging adopts two approaches to model an agent's beliefs. One stream is scenario-based and proceeds by specifying a prediction set $\Omega \subseteq X$ which describes the possible price trajectories. The other stream proceeds by specifying a set $\mathfrak{P} \subseteq \mathcal{P}(X)$ of probability measures (priors) which determines the set of negligible outcomes. We refer to the latter as 
the quasi-sure approach, while the former is usually called the pathwise or pointwise approach. In both cases, the model specification may depend on the agent's market information as well as on her specific modelling assumptions. Changing the set $\Omega$ or $\mathfrak{P}$ can be seen as a natural way to interpolate between different beliefs. One of the principal aims of this paper is to show that both model approaches are equivalent in terms of corresponding FTAPs and superhedging prices.

In order to aggregate trading strategies on different level sets $\Sigma_{t}^{\omega}$ in a measurable way, we always assume in this paper that $\Omega$ is analytic and $\mathfrak{P}$ has the following structure.

Definition 2.1 A set $\mathfrak{P} \subseteq \mathcal{P}(X)$ is said to satisfy the analytic product structure condition (APS) if

$$
\mathfrak{P}=\left\{\mathbb{P}_{0} \otimes \cdots \otimes \mathbb{P}_{T-1}: \mathbb{P}_{t} \text { is an } \mathcal{F}_{t}^{\mathcal{U}} \text {-measurable selector of } \mathfrak{P}_{t}\right\},
$$

where the sets $\mathfrak{P}_{t}(\omega) \subseteq \mathcal{P}\left(X_{1}\right)$ are nonempty, convex and

$$
\operatorname{graph}\left(\mathfrak{P}_{t}\right)=\left\{(\omega, \mathbb{P}): \omega \in X_{t}, \mathbb{P} \in \mathfrak{P}_{t}(\omega)\right\}
$$

is analytic.

This structure facilitates a dynamic programming principle and allows us to essentially paste together one-step results in order to establish their multi-step counterparts.

In order to formulate a fundamental theorem of asset pricing, we need to define the dual objects to trading strategies: the pricing (martingale) measures. Given a set $\mathfrak{P}$ of measures, following Bouchard and Nutz [12], we define

$$
\begin{gathered}
\mathcal{Q}_{\mathfrak{P}, \Phi}:=\left\{\mathbb{Q} \in \mathcal{P}(X): S \text { is an } \mathbb{F}^{\mathcal{U}} \text {-martingale under } \mathbb{Q}, \exists \mathbb{P} \in \mathfrak{P} \text { such that } \mathbb{Q} \ll \mathbb{P},\right. \\
\text { and } \left.\mathbb{E}_{\mathbb{Q}}[\phi]=0, \forall \phi \in \Phi\right\},
\end{gathered}
$$

which in the model-specific case $\mathfrak{P}=\{\mathbb{P}\}$ is simply the familiar set of all martingale measures absolutely continuous with respect to $\mathbb{P}$ and calibrated to $\Phi$. Within the pathwise approach, for a set $\Omega \subseteq X$ and a filtration $\mathbb{F}$, we define

$$
\begin{gathered}
\mathcal{M}_{\Omega, \Phi}^{f}(\mathbb{F}):=\left\{\mathbb{Q} \in \mathcal{P}^{f}(X): S \text { is an } \mathbb{F} \text {-martingale under } \mathbb{Q}, \mathbb{Q}[\Omega]=1,\right. \\
\text { and } \left.\mathbb{E}_{\mathbb{Q}}[\phi]=0, \forall \phi \in \Phi\right\},
\end{gathered}
$$

where $\mathcal{P}^{f}(X)$ denotes the finitely supported Borel probability measures on $(X, \mathcal{B}(X))$. As a general convention, in this paper we interpret the above sub- and superscripts as restrictions on the sets of measures. When we drop some of them it is to indicate that these conditions are not imposed; e.g. $\mathcal{M}_{\Omega}(\mathbb{F})$ denotes all $\mathbb{F}$-martingale measures supported on $\Omega$. Next let

$$
\Omega_{\Phi}^{*}:=\left\{\omega \in \Omega: \exists \mathbb{Q} \in \mathcal{M}_{\Omega, \Phi}^{f}\left(\mathbb{F}^{0}\right) \text { such that } \mathbb{Q}[\{\omega\}]>0\right\}=\bigcup_{\mathbb{Q} \in \mathcal{M}_{\Omega, \Phi}^{f}\left(\mathbb{F}^{0}\right)} \operatorname{supp}(\mathbb{Q})
$$


with the same convention regarding sub- and superscripts as above. We also define

$$
\mathbb{F}^{M}:=\left(\mathcal{F}_{t}^{M}\right)_{t \in\{0,1, \ldots, T\}}, \quad \text { where } \mathcal{F}_{t}^{M}=\bigcap_{\mathbb{Q} \in \mathcal{M}_{\Omega}\left(\mathbb{F}^{0}\right)} \mathcal{F}_{t}^{0} \vee \mathcal{N}^{\mathbb{Q}}\left(\mathcal{F}_{T}^{0}\right)
$$

$\mathcal{N}^{\mathbb{Q}}\left(\mathcal{F}_{T}^{0}\right):=\left\{N \subseteq A \in \mathcal{F}_{T}^{0}: \mathbb{Q}[A]=0\right\}$ and $\mathcal{F}_{t}^{M}$ is the power set of $\Omega$ if $\mathcal{M}_{\Omega}\left(\mathbb{F}^{0}\right)=\emptyset$.

Remark 2.2 Note that $\mathbb{F}^{0} \subseteq \mathbb{F}^{\mathcal{U}} \subseteq \mathbb{F}^{M}$ holds. All these filtrations generate the same martingale measures on $\Omega$ calibrated to $\Phi$, which we denote by $\mathcal{M}_{\Omega, \Phi}$.

For $\mathbb{P} \in \mathcal{P}(X)$, thus $\mathcal{N}^{\mathbb{P}}:=\mathcal{N}^{\mathbb{P}}\left(\mathcal{F}^{\mathcal{U}}\right)$ denotes the collection of its null sets. Likewise, given a family $\mathfrak{P} \subseteq \mathcal{P}(X)$, the collection of its polar sets is given by $\mathcal{N}^{\mathfrak{P}}=\bigcap_{\mathbb{P} \in \mathfrak{P}} \mathcal{N}^{\mathbb{P}}$. We say that a property holds $\mathfrak{P}$-q.s. if it holds outside a $\mathfrak{P}$-polar set.

Let us now introduce a number of notions of arbitrage which will play an important role throughout this article. Over the years, many other notions of arbitrage have been introduced in robust modelling of financial markets. We provide a comprehensive list of these in Sect. 3.2.

Definition 2.3 Fix a filtration $\mathbb{F}$ and sets $\mathfrak{P} \subseteq \mathcal{P}(X)$ and $\Omega \subseteq X$. Recall that semistatic admissible trading strategies are given by $(h, H) \in \mathcal{A}_{\Phi}(\mathbb{F})$.

- 1pA( $(\Omega)$ : A one-point arbitrage (see Riedel [34]) is a strategy $(h, H) \in \mathcal{A}_{\Phi}(\mathbb{F})$ such that $h \cdot \Phi+H \cdot S_{T} \geq 0$ on $\Omega$ with strict inequality for some $\omega \in \Omega$.

- $\mathbf{S A}(\Omega)$ : A strong arbitrage (see Acciaio et al. [1]) is a strategy $(h, H) \in \mathcal{A}_{\Phi}(\mathbb{F})$ such that $h \cdot \Phi+H \cdot S_{T}>0$ on $\Omega$.

- $\mathbf{A}(\mathfrak{P})$ : A $\mathfrak{P}$-quasi-sure arbitrage (see Bouchard and Nutz [12]) is a strategy $(h, H) \in \mathcal{A}_{\Phi}(\mathbb{F})$ with $h \cdot \Phi+H \cdot S_{T} \geq 0 \mathfrak{P}$-q.s. and $\mathbb{P}\left[h \cdot \Phi+H \bullet S_{T}>0\right]>0$ for some $\mathbb{P} \in \mathfrak{P}$. If $\mathfrak{P}=\{\mathbb{P}\}$, a $\mathfrak{P}$-quasi-sure arbitrage is called a $\mathbb{P}$-arbitrage and is denoted $\mathbf{A}(\mathbb{P})$.

When we want to stress the role of the filtration $\mathbb{F}$, we include it as an argument; e.g. we write $\mathbf{S A}(\Omega, \mathbb{F})$. When the filtration is not specified, it is implicitly taken to be $\mathbb{F}^{\mathcal{U}}$. We use a prefix $\mathbf{N}$ to indicate a negation of any of the above notions; e.g. we say that "NSA $(\Omega, \mathbb{F})$ holds" when there does not exist any strong arbitrage strategy for $\Omega$. Likewise $\mathbf{N A}(\mathfrak{P})$ denotes the absence of a $\mathfrak{P}$-quasi-sure arbitrage, etc.

\subsection{Robust fundamental theorem of asset pricing}

The first fundamental theorem of asset pricing characterises the absence of arbitrage in terms of existence of martingale (pricing) measures. In the classical finite-discretetime setting, this refers to the notion of $\mathbb{P}$-arbitrage. However, in a robust setting, there are many possible notions of arbitrage one can consider. If we adopt a strong notion

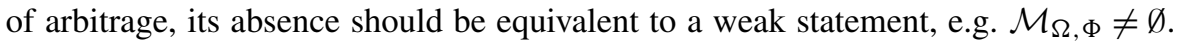
This is often done in the pathwise literature, see Burzoni et al. [13], and leads to a robust (multi-prior) version of the Dalang-Morton-Willinger [22] theorem. 
Theorem 2.4 Let $\mathfrak{P}$ be a set of probability measures satisfying (APS). Then there exist a universally measurable set $\Omega$ of scenarios with $\mathbb{P}[\Omega]=1$ for all $\mathbb{P} \in \mathfrak{P}$ and $a$ filtration $\tilde{\mathbb{F}}$ with $\mathbb{F}^{0} \subseteq \tilde{\mathbb{F}} \subseteq \mathbb{F}^{M}$ such that the following are equivalent:

1) $\mathcal{Q}_{\mathfrak{P}, \Phi} \neq \varnothing$.

2) $\mathbb{P}\left[\Omega_{\Phi}^{*}\right]>0$ for some $\mathbb{P} \in \mathfrak{P}$.

3) $\mathcal{M}_{\Omega, \Phi} \neq \emptyset$.

4) $\Omega_{\Phi}^{*} \neq \emptyset$.

5) $\operatorname{NSA}(\Omega, \tilde{\mathbb{F}})$ holds.

Conversely, for an analytic set $\Omega$, there exists a set $\mathfrak{P}$ satisfying (APS) such that for all $\omega \in \Omega$, there exists $\mathbb{P} \in \mathfrak{P}$ with $\mathbb{P}[\{\omega\}]>0$ and such that 1$)-5)$ are equivalent.

The above result follows from Theorem 2.7 below by setting $\mathcal{S}=\{\Omega\}$. To see its opposite twin, we should adopt a weak notion of arbitrage, its absence thus being equivalent to a strong statement, e.g. for all $\mathbb{P} \in \mathfrak{P}$, there exists $\mathbb{Q} \in \mathcal{Q}_{\mathfrak{P}, \Phi}$ such that $\mathbb{P} \ll \mathbb{Q}$. This route is most often taken in the quasi-sure literature, see Bouchard and Nutz [12], and leads to the following version of the robust FTAP.

Theorem 2.5 Let $\mathfrak{P}$ be a set of probability measures satisfying (APS). Then there exists an analytic set $\Omega$ of scenarios with $\mathbb{P}[\Omega]=1$ for all $\mathbb{P} \in \mathfrak{P}$ such that the following are equivalent:

1) $\operatorname{N1pA}\left(\Omega_{\Phi}^{*}, \mathbb{F}^{\mathcal{U}}\right)$ holds and $\Omega=\Omega_{\Phi}^{*} \mathfrak{P}$-q.s.

2) For all $\mathbb{P} \in \mathfrak{P}$, there exists $\mathbb{Q} \in \mathcal{Q}_{\mathfrak{P}}$, such that $\mathbb{P} \ll \mathbb{Q}$.

3) $N A\left(\mathfrak{P}, \mathbb{F}^{\mathcal{U}}\right)$ holds.

Conversely, if $\Omega$ is an analytic set, then there exists a set $\mathfrak{P}$ of probability measures satisfying (APS) such that for all $\omega \in \Omega$, there exists $\mathbb{P} \in \mathfrak{P}$ with $\mathbb{P}[\{\omega\}]>0$ and such that the following are equivalent:

1) $\operatorname{N1pA}\left(\Omega, \mathbb{F}^{\mathcal{U}}\right)$ holds and $\Omega=\Omega_{\Phi}^{*} \mathfrak{P}$-q.s.

2) For all $\mathbb{P} \in \mathfrak{P}$, there exists $\mathbb{Q} \in \mathcal{Q}_{\mathfrak{P}, \Phi}$ such that $\mathbb{P} \ll \mathbb{Q}$.

3) $N A\left(\mathfrak{P}, \mathbb{F}^{\mathcal{U}}\right)$ holds.

Informally speaking, the set $\Omega$ in the first part of the theorem is constructed as the set of all $\omega \in X$ such that $\Delta S_{t}(\omega)$ lies in the quasi-sure support of

$$
\left\{\mathbb{P} \circ\left(\Delta S_{t}(\omega, \cdot)\right)^{-1}: \mathbb{P} \in \mathfrak{P}_{t-1}(\omega)\right\}
$$

for all $t=1, \ldots, T$. We refer to Sect. 4 for details. Our proof of Theorem 2.5, given in Sect. 4.1, does not rely on the proof of (3) $\Rightarrow$ (2) given in Bouchard and Nutz [12]. Instead we give pathwise arguments. In particular, given $\mathbb{P} \in \mathfrak{P}$ such that $\mathbb{P}\left[\Omega \backslash \Omega_{\Phi}^{*}\right]>0$, we explicitly construct a quasi-sure arbitrage strategy using the universal arbitrage aggregator of Burzoni et al. [13]. This strengthens the results of Theorem 2.7 below. Indeed, using the fact that $\mathfrak{P}$ satisfies (APS), it is possible to select $\mathbb{Q} \in \mathcal{Q}_{\mathfrak{P}, \Phi}$ for each $\mathbb{P} \in \mathfrak{P}$ such that $\mathbb{P} \ll \mathbb{Q}$. Necessarily, the support of each $\mathbb{P}$ is then concentrated on $\Omega_{\Phi}^{*}$. We also remark that in the second part of the theorem, we are 
able to choose the set $\mathfrak{P}$ in such a way that pointwise and $\mathfrak{P}$-quasi-sure equalities are equivalent; this means in particular that $\Omega=\Omega_{\Phi}^{*} \mathfrak{P}$-q.s. holds if and only if $\Omega=\Omega_{\Phi}^{*}$.

Finally, we give our main abstract result. It uses the following notion.

Definition 2.6 In the setup of Definition 2.3, let $\mathcal{S}$ be a set of subsets of $X$.

- $\mathbf{A}(\mathcal{S})$ : An arbitrage de la classe $\mathcal{S}$ (see Burzoni et al. [14]) is a strategy $(h, H)$ in $\mathcal{A}_{\Phi}(\mathbb{F})$ with $h \cdot \Phi+H \cdot S_{T} \geq 0$ on $\Omega$ and $\left\{\omega \in \Omega: h \cdot \Phi+H \cdot S_{T}>0\right\} \supseteq \Gamma$ for some $\Gamma \in \mathcal{S}$.

Our next result establishes a pathwise and probabilistic characterisation of the absence of arbitrage de la classe $\mathcal{S}$. Its proof is presented in Sect. 4.1. Arbitrage de la classe $\mathcal{S}$ allows us to consider many notions of arbitrage at once; see Sect. 3.2. Accordingly, the main result below implies Theorem 2.4 and can be strengthened to imply Theorem 2.5 as will be seen in Sect. 4.

Theorem 2.7 Assume that $\mathfrak{P}$ satisfies $(A P S)$ and $\mathcal{S} \subseteq \mathcal{B}(X)$ is such that

$$
\exists\left(C_{n}\right)_{n \in \mathbb{N}} \subseteq \mathcal{S} \text { such that } \forall C \in \mathcal{S} \exists\left(n_{k}\right)_{k \in \mathbb{N}} \subseteq \mathbb{N} \text { with } \mathbb{1}_{C_{n_{k}}} \uparrow \mathbb{1}_{C} \text { as } k \rightarrow \infty .
$$

Then there exist a co-analytic set $\Omega$ of scenarios such that $\mathbb{P}[\Omega]=1$ for all $\mathbb{P} \in \mathfrak{P}$ and a filtration $\tilde{\mathbb{F}}$ with $\mathbb{F}^{0} \subseteq \tilde{\mathbb{F}} \subseteq \mathbb{F}^{\mathcal{M}}$ such that the following are equivalent:

1) For all $C \in \mathcal{S}$ with $C \subseteq \Omega$, there exists $\mathbb{Q} \in \mathcal{Q}_{\mathfrak{P}, \Phi}$ such that $\mathbb{Q}[C]>0$.

2) For all $C \in \mathcal{S}$ with $C \subseteq \Omega$, there exists $\mathbb{P} \in \mathfrak{P}$ with $\mathbb{P}\left[\Omega_{\Phi}^{*} \cap C\right]>0$.

3) For all $C \in \mathcal{S}$ with $C \subseteq \Omega$, there exists $\mathbb{Q} \in \mathcal{M}_{\Omega, \Phi}$ such that $\mathbb{Q}[C]>0$.

4) $\left\{C \in \mathcal{B}(X): C \subseteq \Omega \backslash \Omega_{\Phi}^{*}\right\} \cap \mathcal{S}=\emptyset$.

5) There is no arbitrage de la classe $\mathcal{S}$ in $\mathcal{A}_{\Phi}(\tilde{\mathbb{F}})$ on $\Omega$.

Conversely, for an analytic set $\Omega$, there exists a set $\mathfrak{P}$ satisfying (APS) such that for all $\omega \in \Omega$, there exists $\mathbb{P} \in \mathfrak{P}$ with $\mathbb{P}[\{\omega\}]>0$ and such that 1$)-5$ ) are equivalent.

Remark 2.8 Condition (2.1) was first stated in Burzoni et al. [14, Corollary 4.30 and the discussion thereafter]. It turns out that for the proof of Theorem 2.7, a weaker condition is sufficient: we only need the properties that

$$
\widetilde{C} \text { is in } \mathcal{B}(X) \text {, where } \widetilde{C}:=\text { the union of all } C \in \mathcal{S} \text { with } C \cap\left(\Omega^{\mathfrak{P}}\right)_{\Phi}^{*} \in \mathcal{N}^{\mathfrak{P}} \text {, }
$$

and

$$
\widetilde{C} \cap\left(\Omega^{\mathfrak{P}}\right)_{\Phi}^{*} \in \mathcal{N}^{\mathfrak{P}}
$$

where we refer to Sect. 4.1 for a formal definition of $\Omega^{\mathfrak{P}}$. Conditions (2.2) and (2.3) are compatibility conditions on $\Omega, \mathcal{S}, \Phi$ and $\mathfrak{P}$. Indeed, they assert that the (likely uncountable) union of "inefficient" subsets of $\Omega^{\mathfrak{P}} \backslash\left(\Omega^{\mathfrak{P}}\right)_{\Phi}^{*}$ (modulo $\mathfrak{P}$-polar sets) stays an "inefficient" subset (modulo $\mathfrak{P}$-polar sets). If this condition is not satisfied for some $\mathfrak{P}$ and $\mathcal{S}$, then there is no reason why a set $\Omega$ for which 2) holds should exist. Take for example a collection $\mathfrak{P}$ having densities and $\mathcal{S}$ a set of singletons in $X$. Then $\mathbb{P}[C]=0$ for any $\mathbb{P} \in \mathfrak{P}$ and $C \in \mathcal{S}$; so the only $\Omega$ which could satisfy 


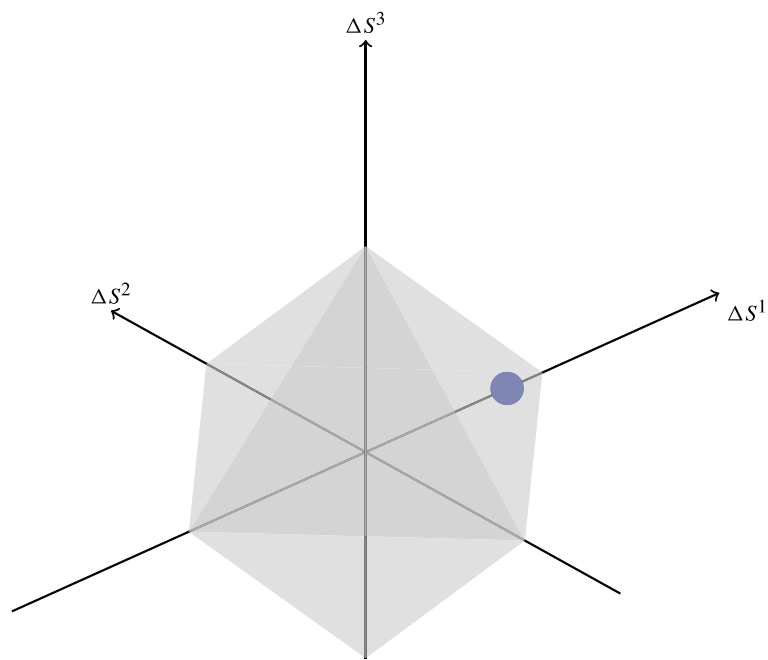

Fig. 2 Construction of a martingale measure $\mathbb{Q} \gg \mathbb{P}$ for $d=3$ : the set $\Omega$ (grey) and the support $\operatorname{supp}\left(\mathbb{P} \circ\left(\Delta S_{1}\right)^{-1}\right)$ (blue)

2) is the empty set. We note that when $\mathcal{S}=\{C \subseteq X: C$ open $\}$, then (2.2) is always satisfied and (2.3) is satisfied as soon as $X$ is separable. However, conditions (2.2) and (2.3) may be hard to verify in general, which is why we provide (2.1) as an easier sufficient condition. Finally, we remark that it is not straightforward to show that $\mathcal{S}=\{C: \mathbb{P}[C]>0$ for some $\mathbb{P} \in \mathfrak{P}\}$ corresponding to NA(P) satisfies (2.3), which is why we give a direct proof of Theorem 2.5 in Sect. 4 .

We note that the set $\Omega$ cannot be taken to be analytic in general. The implications 1) $\Rightarrow 2) \Rightarrow 3) \Rightarrow 4) \Rightarrow 5$ ) follow directly from the definitions. Apart from measurability considerations regarding $\Omega$, the equivalence of 3)-5) essentially follows from Burzoni et al. [14]. Furthermore, given an analytic set $\Omega$, we simply define $\mathfrak{P}$ as all the finitely supported probability measures on $\Omega$. The analyticity of $\Omega$ then implies (APS) of $\mathfrak{P}$. We then also have $\mathcal{Q}_{\mathfrak{P}, \Phi}=\mathcal{M}_{\Omega, \Phi}^{f}$ and equivalence of 1) and 3)-5) follows from Burzoni et al. [13]. In this context, the essential connection we make is the combination of pathwise and quasi-sure criteria as stated in 2): for every $C \in \mathcal{S}$, the pathwise efficient subset $\Omega_{\Phi}^{*} \cap C$ is required to be "seen" by at least one measure $\mathbb{P}$ in the set $\mathfrak{P}$.

The main difficulty of the proof of Theorem 2.7 is then to show the implication $5) \Rightarrow 1$ ), where one needs to establish existence of martingale measures $\mathbb{Q} \in \mathcal{Q}_{\mathfrak{P}, \Phi}$ which are compatible with $\Omega$ and $\mathcal{S}$ in the sense of 1). This, modulo measurable selection arguments, is achieved by finding an element $\mathbb{P} \in \mathfrak{P}_{t}(\omega)$ such that zero is in the relative interior of the support of $\mathbb{P} \circ\left(\Delta S_{t+1}\right)^{-1}$. Indeed, let us explain the main idea of the proof of 5) $\Rightarrow 1$ ) based on the following example. Assume $T=1, d=3$, $\Phi=0, \mathcal{S}=\{\Omega\}$ and the set $\Omega^{*}$ is given by the grey polyhydron in Fig. 2. Assume that the support of $\mathbb{P} \circ\left(\Delta S_{1}\right)^{-1}$ for a given measure $\mathbb{P} \in \mathfrak{P}_{0}$ is given by the blue dot (see Fig. 2). Then as $0 \in \operatorname{ri}\left(\Omega^{*}\right)$, we can find three additional points in $\Omega^{*}$ such that zero is in the relative interior of the convex hull of the four points. By the definition 


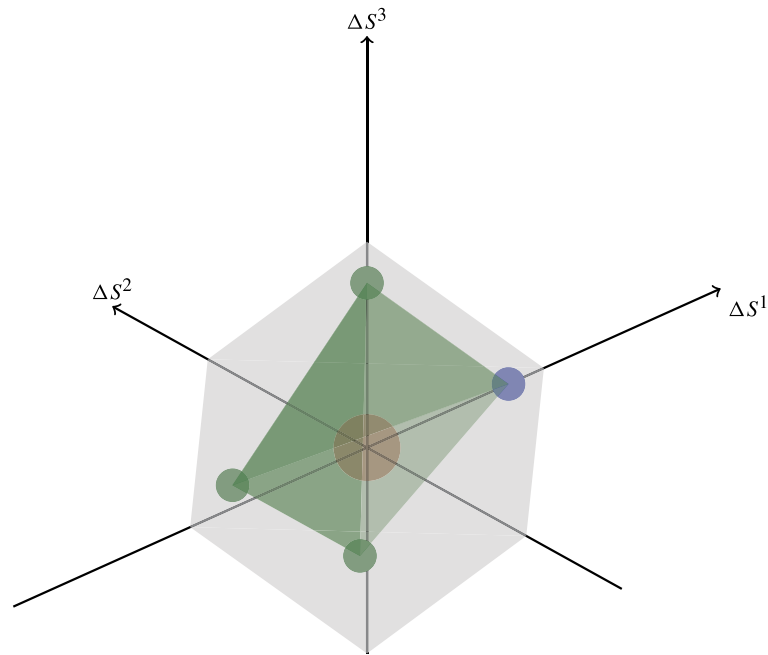

Fig. 3 Construction of a martingale measure $\mathbb{Q} \gg \mathbb{P}$ for $d=3$ : finding a measure $\tilde{\mathbb{P}}$ such that $\mathrm{NA}(\tilde{\mathbb{P}})$ and $\tilde{\mathbb{P}} \gg \mathbb{P}$ hold

of $\Omega$, the three additional points are in the support of some measures in $\mathfrak{P}_{0}$, which we call $\mathbb{P}_{1}, \mathbb{P}_{2}, \mathbb{P}_{3}$ in $\mathfrak{P}_{0}$. As $\mathfrak{P}_{0}$ is convex, it follows that

$$
\tilde{\mathbb{P}}:=\frac{\mathbb{P}_{1}+\mathbb{P}_{2}+\mathbb{P}_{3}+\mathbb{P}}{4}
$$

is an element of $\mathfrak{P}_{0}$ as well, as visualised in Fig. 3. Since 0 is in the relative interior of the support of $\tilde{\mathbb{P}}$, one can now use results from Rokhlin [36] to find a martingale measure $\mathbb{Q} \approx \tilde{\mathbb{P}}$, in particular $\mathbb{P} \ll \mathbb{Q}$.

Note that this argument fundamentally relies on the convexity of $\mathfrak{P}_{t}$. The analytic product structure assumption then grants suitable measurability for the concatenation procedure in the multi-period case.

\subsection{Robust superhedging theorem}

In this section, we focus on the key result which characterises superhedging prices: the pricing-hedging duality, or the superhedging theorem. As before, we compare pathwise and quasi-sure superhedging approaches as extensions of the classical model-specific result; see Föllmer and Schied [27, Theorem 5.30].

For a set $\Omega \subseteq X$, we denote the pathwise superhedging price on $\Omega$ by

$$
\begin{array}{r}
\pi_{\Omega}(g):=\inf \left\{x \in \mathbb{R}: \exists(h, H) \in \mathcal{A}_{\Phi}\left(\mathbb{F}^{\mathcal{U}}\right)\right. \text { such that } \\
\left.x+h \cdot \Phi+\left(H \cdot S_{T}\right) \geq g \text { on } \Omega\right\}
\end{array}
$$

and the $\mathfrak{P}$-q.s. superhedging price by

$$
\begin{aligned}
\pi^{\mathfrak{P}}(g):=\inf \{x \in \mathbb{R}: & \exists(h, H) \in \mathcal{A}_{\Phi}\left(\mathbb{F}^{\mathcal{U}}\right) \text { such that } \\
& \left.x+h \cdot \Phi+\left(H \bullet S_{T}\right) \geq g \mathfrak{P} \text {-q.s. }\right\} .
\end{aligned}
$$


Take an analytic set $\Omega$ such that $\mathbb{P}\left[\Omega_{\Phi}^{*}\right]=1$ for all $\mathbb{P} \in \mathfrak{P}$. Using the superhedging theorems of Bouchard and Nutz [12] and Burzoni et al. [13], it is immediate that we have for all upper semianalytic $g$ the relationships

$$
\sup _{\mathbb{Q} \in \mathcal{M}_{\Omega, \Phi}} \mathbb{E}_{\mathbb{Q}}[g]=\pi_{\Omega_{\Phi}^{*}}(g) \geq \pi^{\mathfrak{P}}(g)=\sup _{\mathbb{Q} \in \mathcal{Q}_{\mathfrak{P}, \Phi}} \mathbb{E}_{\mathbb{Q}}[g] .
$$

The above inequality is strict in general. An easy example where one can see this is to take $d=T=S_{0}=1, \Phi=0, g\left(S_{1}\right)=\mathbb{1}_{\left\{S_{1}=0\right\}}$ and $\mathfrak{P}=\left\{\left.\frac{1}{2} \lambda\right|_{[0,2]}\right\}$, where $\left.\lambda\right|_{[0,2]}$ denotes Lebesgue measure on $[0,2]$. Then $\Omega=\Omega^{*}=[0,2]$ and the pathwise superhedging price is equal to $1 / 2$, while the quasi-sure superhedging price is equal to zero. In fact, to link the superhedging and pathwise formulations, we have to choose a specific set $\Omega_{g}^{\mathfrak{P}}$ which depends not only on $\mathfrak{P}$, but also on $g$. We determine this set $\Omega_{g}^{\mathfrak{P}}$ by reducing to superhedging under a fixed measure $\mathbb{P}^{g}$ as stated in the following theorem.

Theorem 2.9 Let $\mathfrak{P}$ be a set of probability measures satisfying (APS). Assume NA(P) holds and let $g: X \rightarrow \mathbb{R}$ be an upper semianalytic function. Then there exist a measure $\mathbb{P}^{g}=\mathbb{P}_{0}^{g} \otimes \cdots \otimes \mathbb{P}_{T-1}^{g}$ and an $\mathcal{F}^{\mathcal{U}}$-measurable set $\Omega_{g}^{\mathfrak{P}}$ with $\mathbb{P}\left[\Omega_{g}^{\mathfrak{P}}\right]=1$ for all $\mathbb{P} \in \mathfrak{P}$ such that

$$
\sup _{\mathbb{Q} \in \mathcal{M}_{\Omega_{g}^{\mathfrak{P}}, \Phi}} \mathbb{E}_{\mathbb{Q}}[g]=\pi_{\left(\Omega_{g}^{\mathfrak{P}}\right)_{\Phi}^{*}}(g)=\pi^{\mathbb{P}^{g}}(g)=\pi^{\mathfrak{P}}(g)=\sup _{\mathbb{Q} \in \mathcal{Q}_{\mathfrak{P}, \Phi}} \mathbb{E}_{\mathbb{Q}}[g] .
$$

Conversely, let $\Omega$ be an analytic subset of $X$ with $\Omega_{\Phi}^{*} \neq \emptyset$ and $g: X \rightarrow \mathbb{R}$ upper semianalytic. For any set $\mathfrak{P} \subseteq \mathcal{P}(X)$ which satisfies $(A P S)$ and $\mathcal{N}^{\mathfrak{P}}=\mathcal{N}^{\mathcal{M}}{ }_{\Omega, \Phi}^{f}$, we have

$$
\sup _{\mathbb{Q} \in \mathcal{M}_{\Omega, \Phi}^{f}} \mathbb{E}_{\mathbb{Q}}[g]=\pi_{\Omega_{\Phi}^{*}}(g)=\pi^{\mathfrak{P}}(g)=\sup _{\mathbb{Q} \in \mathcal{Q}_{\mathfrak{P}, \Phi}} \mathbb{E}_{\mathbb{Q}}[g]
$$

In both cases, if the value is finite, it is attained by a superhedging strategy $(h, H)$ in $\mathcal{A}_{\Phi}\left(\mathbb{F}^{\mathcal{U}}\right)$.

We make three remarks about the above results and postpone the proof to Sect. 4.2.

Remark 2.10 The first part of Theorem 2.9 lets us interpret the robust superhedging price $\pi^{\mathfrak{P}}(g)$ as the classical superreplication price $\pi^{\mathbb{P}^{g}}(g)$ under an "extremal" measure $\mathbb{P}^{g}$. Determining such measures $\mathbb{P}^{g}$ is not straightforward in general. In the one-period case and for a continuous $g$, we can use the arguments in the proof of Lemma 4.11 to see that any measure $\tilde{\mathbb{P}}$ for which the one-step support of $\left.\tilde{\mathbb{P}} \circ\left(\Delta S_{T}(\omega, \cdot)\right)^{-1}\right)$ and the one-step quasi-sure support of $\left\{\mathbb{P} \circ\left(\Delta S_{T}(\omega, \cdot)\right)^{-1}: \mathbb{P} \in \mathfrak{P}_{T-1}(\omega)\right\}$ agree can be chosen. To extend this result to the general case, certain continuity properties of the maps $\omega \mapsto \mathfrak{P}_{t}(\omega)$ have to be guaranteed; we refer to Carassus et al. [17, Proposition 3.7] for a sufficient condition. 
Remark 2.11 As in Sect. 2.2, the corresponding result going from the pointwise to the quasi-sure setup in the second part of Theorem 2.9 is more straightforward: we only have to define the set $\mathfrak{P}$ of measures in such a way that it "sees" all the points $\omega \in \Omega_{\Phi}^{*}$ (i.e., all the points $\omega \in \Omega$ for which the pointwise superhedging constraint holds). This can be achieved by essentially setting $\mathfrak{P}=\left\{\delta_{\omega}: \omega \in \Omega_{\Phi}^{*}\right\}$. More generally, the condition $\mathcal{N}^{\mathfrak{P}}=\mathcal{N}^{\mathcal{M}_{\Omega, \Phi}^{f}}$ is sufficient. We also remark that $\mathcal{N}^{\mathcal{M}}{ }_{\Omega, \Phi}^{f}=\mathcal{N}^{\mathcal{M} \Omega, \Phi}$ holds. This follows from results in Burzoni et al. [13, proofs of Lemmas 2 and 4].

Remark 2.12 In contrast to Burzoni et al. [13, Theorem 2], we only consider upper semianalytic claims $g$, while [13, Theorem 2] holds for the larger class of analytically measurable claims. The benefit of this is that we can restrict to $\mathbb{F}^{\mathcal{U}}$-predictable trading strategies for superhedging instead of using the a priori harder to characterise filtration $\mathbb{F}^{\mathrm{pr}}$ which is needed for the duality results in [13]. This fact allows a more direct comparison with results from Bouchard and Nutz [12], where also $\mathbb{F}^{\mathcal{U}}$-predictable strategies are used.

\section{Complementary results on superhedging and arbitrage}

\subsection{Extension of pathwise superhedging from $\Omega^{*}$ to $\Omega$}

The preceding results show that quasi-sure and pathwise superhedging are essentially equivalent. As $\mathfrak{P}$-q.s. superhedging strategies might be difficult to compute and implement in practice, it might be preferable to work on a prediction set $\Omega$ using pathwise arguments. Given that determining $\Omega^{*}$ is computationally expensive as well, the quantity of interest is then the superhedging price on $\Omega$ and not on $\Omega^{*}$ seen in the duality results in Sect. 2.3. Thus, we should like to find sufficient conditions under which the superhedging strategy associated with $\pi_{\Omega_{\Phi}^{*}}(g)$ can be extended to $\Omega$ without any additional cost. The intuition is that as $\Omega \backslash \Omega^{*}$ describes non-efficient beliefs, we should be able to superhedge $g$ on this set by implementing an arbitrage strategy. It turns out that this intuition is not true in general. Indeed, we run into problems regarding measurability of these arbitrage strategies, which means that this procedure only works in special cases.

To simplify the analysis, throughout this section only, we assume that $\Phi=0$ and $\omega \mapsto S_{t}(\omega)$ is continuous. The latter is satisfied e.g. when $\omega \mapsto S_{t}(\omega)$ is the coordinate mapping, i.e., $S_{t}(\omega)=\omega_{t}$. In order to give some intuition and to identify necessary conditions for the sets $\Omega, \Omega^{*}$ and the function $g$, we first give two counterexamples.

Example 3.1 Let $d=1, T=1$ and $(\Omega, \mathcal{F})=\left(\mathbb{R}_{+} \backslash\{0\}, \mathcal{B}\left(\mathbb{R}_{+} \backslash\{0\}\right)\right)$. We set $S_{0}=2$ and $S_{1}(\omega)=2+\omega$. Then $\Omega^{*}=\emptyset$ and trivially

$$
\begin{gathered}
\pi_{\Omega^{*}}(1)=\inf \left\{x \in \mathbb{R}: \exists H \in \mathbb{R}^{d} \text { such that } x+H \cdot S_{T} \geq 1 \text { on } \Omega^{*}\right\}=-\infty, \\
\pi_{\Omega}(1)=\inf \left\{x \in \mathbb{R}: \exists H \in \mathbb{R}^{d} \text { such that } x+H \cdot S_{T} \geq 1 \text { on } \Omega\right\}=1 .
\end{gathered}
$$

Thus we have to assume that $\Omega^{*} \cap \Sigma_{t}^{\omega} \neq \varnothing$ in the remainder of this section. We also note that here $\mathbf{S A}(\Omega)$ holds, while $\mathbf{U S A}(\Omega)$ does not; see Sect. 3.2. 
Example 3.2 Let $d=2, T=1$ and $\Omega=((2, \infty) \times[0, \infty)) \cup(\{2\} \times[0,7])$ and $\mathcal{F}=\mathcal{B}(\Omega)$. We set $S_{0}=(2,2)$ and $S_{1}(\omega)=\omega$. In particular, $\Delta S_{1}(\Omega)$ is not a closed set. Note that $\Omega^{*}=\{2\} \times[0,7]$. Now introduce the claim

$$
g\left(S^{1}, S^{2}\right)=\Delta S_{1}^{2} \mathbb{1}_{\left\{\Delta S_{1}^{2} \leq 5\right\}}+5\left(\Delta S_{1}^{2}-4\right) \mathbb{1}_{\left\{\Delta S_{1}^{2}>5\right\}} .
$$

It is easy to see that $\pi_{\Omega^{*}}(g)=0$ and any trading strategy $H_{1}=\left(H_{1}^{1}, 1\right)$ with $H_{1}^{1} \in \mathbb{R}$ is a superhedging strategy. We now claim that we cannot extend superhedging to $\Omega \backslash \Omega^{*}$ with initial capital zero. For this we show that even for initial capital 1 , there exist no superreplication strategies on $\Omega$. Indeed, for this we should need

$$
1+H_{1}^{1} \Delta S_{1}^{1}+H_{1}^{2} \Delta S_{1}^{2} \geq 5\left(\Delta S_{1}^{2}-4\right) \quad \text { on }\left\{\Delta S_{1}^{1}>0, \Delta S_{1}^{2}>5\right\},
$$

which is equivalent to

$$
H_{1}^{1} \geq \frac{\left(5-H_{1}^{2}\right) \Delta S_{1}^{2}-21}{\Delta S_{1}^{1}} .
$$

As $H_{1}^{2} \in[4 / 5,3 / 2]$, this means that $H_{1}^{1} \rightarrow \infty$ if $\Delta S_{1}^{1}$ is arbitrarily close to 0 and $\Delta S_{1}^{2}$ is sufficiently large. Even if we look at

$$
\Omega=([2+\varepsilon, \infty) \times[0, \infty)) \cup(\{2\} \times[0,7])
$$

for some positive $\varepsilon$, taking $\Delta S_{1}^{2}$ arbitrarily large still leads to non-existence of superhedging strategies. In conclusion, we only consider compact sets $\Omega \cap \Sigma_{t}^{\omega}$ in the rest of this section, on which "arbitrage strategies are effective for superhedging" in a sense defined below. Furthermore, modifying the function $g$ on $\Omega \backslash \Omega^{*}$ in the above example, we can easily construct situations in which $\pi_{\Omega}(g) \neq \pi_{\Omega^{*}}(g)$ for discontinuous payoffs $g$. In conclusion, we also assume that $g$ is continuous in this section.

We can also modify this example so that $\Delta S_{1}(\Omega)$ is closed and there is no attainment of superreplication strategies for $\pi_{\Omega}(g)$. We stress that this is a fundamental difference to the case $\Omega=\Omega^{*}$, where attainment is always given (see Theorem 2.9). Namely, take

$$
\Omega=\left\{(x, y) \in \mathbb{R}^{2}: x \in[2, \infty), 0 \leq y \leq 5+\sqrt{x}\right\}
$$

with the other elements unchanged. Note that $\Omega^{*}$ did not change. Repeating the arguments above and looking at $\Delta S_{1}^{1}=1 / n$ and $\Delta S_{1}^{2}=5+1 / \sqrt{n}$, we find

$$
H_{1}^{1} \geq n\left(20+\frac{5}{\sqrt{n}}-20-\frac{1}{\sqrt{n}}\right)=n \frac{4}{\sqrt{n}} \longrightarrow \infty
$$

for $n \rightarrow \infty$.

As we have seen in the examples above, it is necessary in general to assume that $\Omega^{*} \cap \Sigma_{t}^{\omega} \neq \varnothing, \omega \mapsto g(\omega)$ is continuous and that $\Omega$ is compact as well as "well suited for superhedging by arbitrage strategies". We first address the second point and show continuity of the one-step superhedging prices $\omega \mapsto \pi_{t, \Omega^{*}}(g)(\omega)$, which are defined via a dynamic programming approach. 
Definition 3.3 For a Borel-measurable $g: X \rightarrow \mathbb{R}$, we define the one-step superhedging prices

$$
\begin{aligned}
& \pi_{T, \Omega^{*}}(g)(\omega):=g(\omega), \\
& \pi_{t, \Omega^{*}}(g)(\omega):=\inf \left\{x \in \mathbb{R}: \exists H \in \mathbb{R}^{d}\right. \text { such that } \\
& \left.x+H \Delta S_{t+1}(v) \geq \pi_{t+1, \Omega^{*}}(g)(v), \forall v \in \Sigma_{t}^{\omega} \cap \Omega^{*}\right\},
\end{aligned}
$$

where $t=0,1, \ldots, T-1$.

A sufficient condition for continuity of $\omega \mapsto \pi_{t, \Omega^{*}}(g)(\omega)$ was identified in Carassus et al. [17] and relies on the following assumption.

Assumption 3.4 The sets $\Sigma_{t}^{\omega} \cap \Omega^{*} \neq \emptyset$ and $\Sigma_{t}^{\omega} \cap \Omega$ are compact for all $\omega \in \Omega$ and $t=0,1, \ldots, T-1$. Furthermore, for all $t=0,1, \ldots, T-1$, the correspondences $\omega \rightarrow S_{t+1}\left(\Sigma_{t}^{\omega} \cap \Omega^{*}\right)$ and $\omega \rightarrow \operatorname{span}\left(S_{t+1}\left(\Sigma_{t}^{\omega} \cap \Omega^{*}\right)-S_{t}(\omega)\right)$ are continuous and upper hemicontinuous, respectively, from $\left(\Omega, d_{t}^{S}\right)$ to the subsets of $\mathbb{R}^{d}$ endowed with the Hausdorff distance, and where

$$
d_{t}^{S}(\omega, \tilde{\omega}):=\max _{s=0,1, \ldots, t}\left|S_{S}(\omega)-S_{S}(\tilde{\omega})\right| .
$$

We refer to Carassus et al. [17, Sect. 3] for a discussion and examples of sets $\Omega$ satisfying Assumption 3.4. The following lemma now follows from a direct application of [17, Proposition 3.7]. ${ }^{1}$

Lemma 3.5 Let $\omega \mapsto g(\omega)$ be continuous. Under Assumption 3.4, the one-step superhedging prices $\omega \mapsto \pi_{t, \Omega^{*}}(g)(\omega)$ are continuous for all $t=0,1, \ldots, T-1$.

Secondly, Example 3.2 also shows that it is important to identify the subset of $\Sigma_{t}^{\omega} \cap \Omega$ on which "arbitrage strategies are ineffective for superhedging" in the following sense.

Definition 3.6 We denote by $\operatorname{proj}_{\Delta S_{t+1}\left(\Sigma_{t}^{\omega} \cap \Omega^{*}\right)}\left(\Delta S_{t+1}(v)\right)$ the orthogonal projection of $\Delta S_{t+1}(v)$ onto the linear subspace spanned by $\Delta S_{t+1}\left(\Sigma_{t}^{\omega} \cap \Omega^{*}\right)$ and define the set $A_{t}^{\omega}$ as the collection of all $v \in \Sigma_{t}^{\omega} \cap \Omega$, for which $\operatorname{proj}_{\Delta S_{t+1}\left(\Sigma_{t}^{\omega} \cap \Omega^{*}\right)}\left(\Delta S_{t+1}(v)\right)$ is not an element of $\Delta S_{t+1}\left(\Sigma_{t}^{\omega} \cap \Omega^{*}\right)$.

For an illustration of the set $A_{t}^{\omega}$, see Fig. 4. We now state an assumption ensuring compatibility of $A_{t}^{\omega}$ and $\Sigma_{t}^{\omega} \cap \Omega^{*}$.

Assumption 3.7 For each level set, the following is true: If a sequence of points $\left(v_{n}\right) \subseteq A_{t}^{\omega}$ converges to a point $v \in \operatorname{span}\left(\Delta S_{t+1}\left(\Sigma_{t}^{\omega} \cap \Omega^{*}\right)\right)$, then necessarily $v \in \Delta S_{t+1}\left(\Sigma_{t}^{\omega} \cap \Omega^{*}\right)$.

\footnotetext{
${ }^{1}$ We note that the result in [17] is missing the assumption on $\operatorname{span}\left(S_{t+1}\left(\Sigma_{t}^{\omega} \cap \Omega^{*}\right)-S_{t}(\omega)\right)$ as pointed out to us by Sergey Smirnov.
} 
Fig. 4 Example 3.2 with notation from Definition 3.6

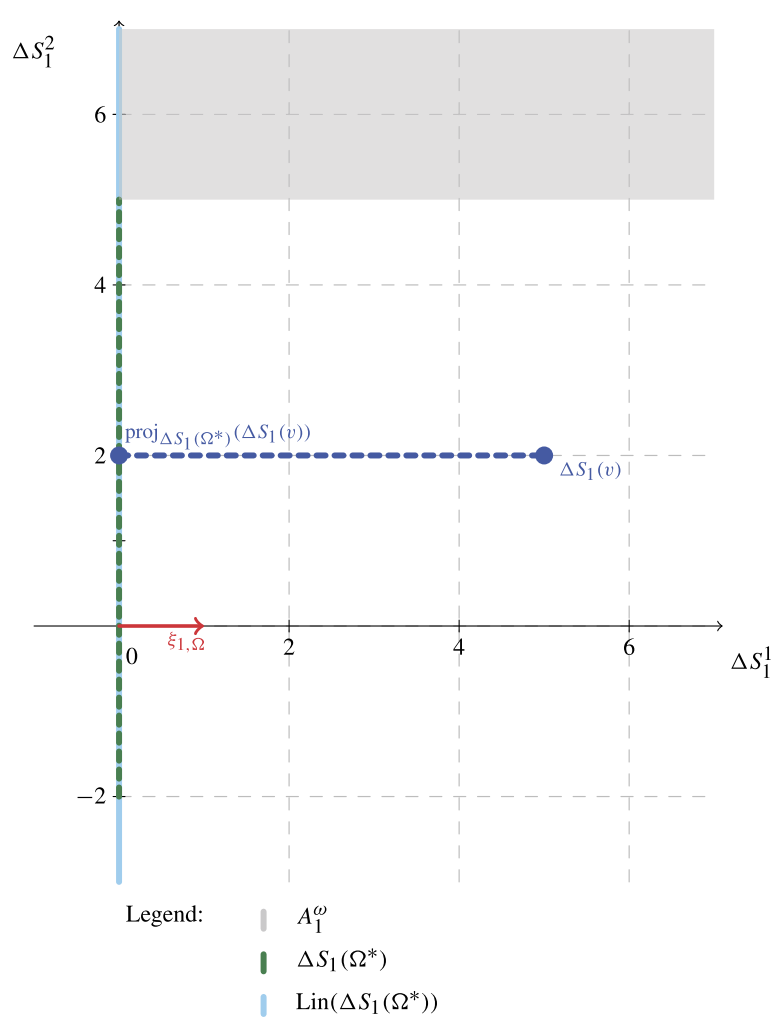

Alas, it turns out that while Assumptions 3.4 and 3.7 are sufficient to establish the equality $\pi_{\Omega}(g)=\pi_{\Omega^{*}}(g)$ for $d=2$, it is not so for $d>2$. This is linked with the notion of standard separators introduced in Burzoni et al. [13], which are measurable selectors of pointwise arbitrage strategies. We refer the reader to [13, proof of Lemma 1] and the discussion therein for a detailed definition. Here we formulate an example in which the existence of two standard separators together with the measurability constraint on $H_{1}$ implies $\pi_{\Omega}(g)>\pi_{\Omega^{*}}(g)$.

Example 3.8 Let $d=3, T=1$ and $(\Omega, \mathcal{F})=\left(\mathbb{R}_{+}, \mathcal{B}\left(\mathbb{R}_{+}\right)\right)$. We take initial stock prices $\left(S_{0}^{1}, S_{0}^{2}, S_{0}^{3}\right)=(2,2,2)$ and

$$
\begin{aligned}
& S_{1}^{1}(\omega)= \begin{cases}2 & \text { if } \omega \in \mathbb{R}_{+} \backslash \mathbb{Q}, \\
2.5-\omega & \text { if } \omega \in \mathbb{Q} \cap[1 / 2, \infty), \\
4 & \text { if } \omega \in \mathbb{Q} \cap[0,1 / 2),\end{cases} \\
& S_{1}^{2}(\omega)= \begin{cases}\omega & \text { if } \omega \in \mathbb{R}_{+} \backslash \mathbb{Q}, \\
0 & \text { if } \omega \in \mathbb{Q} \cap[1 / 2, \infty), \\
2 & \text { if } \omega \in \mathbb{Q} \cap[0,1 / 2),\end{cases} \\
& S_{1}^{3}(\omega)= \begin{cases}2 & \text { if } \omega \in \mathbb{R}_{+} \backslash \mathbb{Q}, \\
2 & \text { if } \omega \in \mathbb{Q} \cap[1 / 2, \infty), \\
2+\omega & \text { if } \omega \in \mathbb{Q} \cap[0,1 / 2) .\end{cases}
\end{aligned}
$$



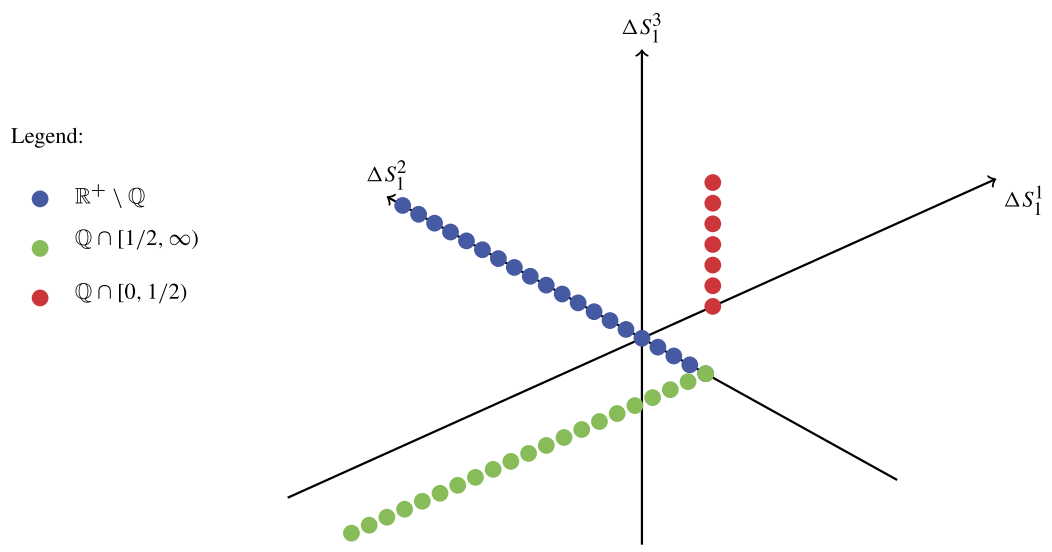

Fig. 5 Range of $S_{1}^{1}, S_{1}^{2}$ and $S_{1}^{3}$ in Example 3.8

For an illustration of the range of $S_{1}^{1}, S_{1}^{2}$ and $S_{1}^{3}$, see Fig. 5. Then $\Omega^{*}=\mathbb{R}_{+} \backslash \mathbb{Q}$, and using the notation of Burzoni et al. [13, proof of Lemma 1], the standard separators are given by $\xi_{0, A_{0}}=(0,0,1)$ and $\xi_{0, A_{1}}=(-1,0,0)$. Next we define

$$
g\left(S^{1}, S^{2}, S^{3}\right)= \begin{cases}\left(\Delta S_{1}^{2}+\left|\Delta S_{1}^{1}\right|+\left|\Delta S_{1}^{3}\right|\right)^{-} & \text {if } \Delta S_{1}^{2} \leq 0 \\ \left(\Delta S_{1}^{2}-\left|\Delta S_{1}^{1}\right|-\left|\Delta S_{1}^{3}\right|\right)^{+} & \text {if } \Delta S_{1}^{2}>0 .\end{cases}
$$

We note that for $\Delta S_{1}^{1}=\Delta S_{1}^{3}=0$, we have $g(S)=\Delta S_{1}^{2}$. So in particular to hedge $g$ on $\Omega^{*}$, we need initial capital $\pi_{\Omega^{*}}(g)=0$ and any hedging strategy satisfies $H_{1}=\left(H_{1}^{1}, 1, H_{1}^{3}\right)$ for $H_{1}^{1}, H_{1}^{3} \in \mathbb{R}$. For any such strategy to also superhedge on $\mathbb{Q} \cap[1 / 2, \infty)$ with initial capital $1 / 2, H_{1}^{1}$ must satisfy in particular

$$
1 / 2+H_{1}^{1} \Delta S_{1}^{1}-2 H_{1}^{2} \geq 0 \quad \text { for } \Delta S_{1}^{1} \leq-2,
$$

so $H_{1}^{1} \leq-3 / 4$ as $H_{1}^{2} \in[1,3 / 2]$. Finally, extending superhedging $g$ on $\mathbb{Q} \cap[0,1 / 2)$ gives the constraint

$$
1 / 2+2 H_{1}^{1}+H_{1}^{3} \Delta S_{1}^{3} \geq 0
$$

Taking $\Delta S_{1}^{3}=0$ gives $H_{1}^{1} \geq-1 / 4$, a contradiction. Thus Assumption 3.9 below imposes that all one-point arbitrages can be reduced to a single standard separator.

Note that Example 3.8 can be easily altered to make $\Delta S_{t+1}\left(\Omega \cap \Sigma_{t}^{\omega}\right)$ convex and compact by adding additional points. For clarity of exposition, we have refrained from doing this, but we conclude that Assumptions 3.4 and 3.7 are not sufficient for $d>2$. We have to add a last assumption which guarantees measurability of the corresponding universal arbitrage aggregator. Intuitively, it states that locally, i.e., for $t=0,1, \ldots, T-1$ and every $\omega \in \Omega$, there exists at most one arbitrageable direction 
of the evolution of assets $S$, so that the first standard separator is already the universal arbitrage aggregator.

Assumption 3.9 For all $\omega \in \Omega$ and $t=0,1, \ldots, T-1$, we have $\xi_{t+1, \Omega \cap \Sigma_{t}^{\omega}}=H_{t}^{*}$, where $H^{*}$ is the universal arbitrage aggregator of Burzoni et al. [13] for the set $\Sigma_{t}^{\omega} \cap \Omega$.

Theorem 3.10 Suppose $\Phi=0$ and $X \ni \omega \mapsto S_{t}(\omega)$ is continuous for $t=1, \ldots, T$. For an analytic set $\Omega \subseteq X$ satisfying Assumptions 3.4, 3.7 and 3.9, the superhedging duality of Burzoni et al. [13] extends from $\Omega^{*}$ to $\Omega$ for all continuous $g: X \rightarrow \mathbb{R}$, i.e., we have

$$
\begin{aligned}
& \sup \left\{\mathbb{E}_{\mathbb{Q}}(g): \mathbb{Q} \in \mathcal{M}_{\Omega}^{f}\right\} \\
& =\inf \left\{x \in \mathbb{R}: \exists H \in \mathcal{H}\left(\mathbb{F}^{\mathcal{U}}\right) \text { such that } x+H \cdot S_{T} \geq g \text { on } \Omega^{*}\right\} \\
& =\inf \left\{x \in \mathbb{R}: \exists H \in \mathcal{H}\left(\mathbb{F}^{\mathcal{U}}\right) \text { such that } x+H \cdot S_{T} \geq g \text { on } \Omega\right\} .
\end{aligned}
$$

Proof We argue by backward induction over $t=0, \ldots, T-1$. Let us fix $\omega \in \Omega$. We assume $\Sigma_{t}^{\omega} \cap \Omega^{*} \neq \emptyset$ and $\left(\Sigma_{t}^{\omega} \cap \Omega\right) \backslash\left(\Sigma_{t}^{\omega} \cap \Omega^{*}\right) \neq \emptyset$, otherwise the claim is trivial. We first look at the case where $\operatorname{proj}_{\Delta S_{t+1}\left(\Sigma_{t}^{\omega} \cap \Omega^{*}\right)}\left(\Delta S_{t+1}(v)\right)$ is an element of $\Delta S_{t+1}\left(\Sigma_{t+1}^{\omega} \cap \Omega^{*}\right)$, i.e., there exists $v^{\prime} \in \Sigma_{t}^{\omega} \cap \Omega^{*}$ such that

$$
\operatorname{proj}_{\Delta S_{t+1}\left(\Sigma_{t}^{\omega} \cap \Omega^{*}\right)}\left(\Delta S_{t+1}(v)\right)=\Delta S_{t+1}\left(v^{\prime}\right)
$$

Note that by Assumption 3.9, the standard separator $\xi_{t+1, \Omega \cap \Sigma_{t}^{\omega}}$ is orthogonal to $\operatorname{span}\left(\Delta S_{t+1}\left(\Sigma_{t}^{\omega} \cap \Omega^{*}\right)\right)$. By the definition of the superhedging price on $\Omega$, there exists an $\mathcal{F}_{t}^{\mathcal{U}}$-measurable strategy $H_{t+1}$ such that

$$
\pi_{t, \Omega^{*}}(g)\left(v^{\prime}\right)+H_{t+1}(\omega) \Delta S_{t+1}\left(v^{\prime}\right) \geq \hat{\pi}_{t+1}(g)\left(v^{\prime}\right) \quad \text { for all } v^{\prime} \in \Sigma_{t}^{\omega} \cap \Omega^{*},
$$

where we can assume that $H_{t+1}(\omega) \in \operatorname{span}\left(\Delta S_{t+1}\left(\Sigma_{t}^{\omega} \cap \Omega^{*}\right)\right)$ without loss of generality. Now we fix $v \in \Sigma_{t}^{\omega} \cap \Omega$ and $v^{\prime}$ as the corresponding orthogonal projection. Let $\varepsilon>0$. As $\pi_{t+1, \Omega^{*}}(g)$ is uniformly continuous on $\Omega \cap \Sigma_{t}^{\omega}$, we can use Vanderbei [40, Theorem 1] (in connection with Tietze's extension theorem to extend the domain to a convex set) in order to find $\delta>0$ such that

$$
\varepsilon+\pi_{t+1, \Omega^{*}}(g)\left(v^{\prime}\right)+\frac{S_{t+1}(v)-S_{t+1}\left(v^{\prime}\right)}{\delta / \varepsilon} \xi_{t+1, \Sigma_{t}^{\omega} \cap \Omega}(v) \geq \pi_{t+1, \Omega^{*}}(g)(v),
$$

where $\delta$ is chosen such that for all $w, \tilde{w} \in \Sigma_{t}^{\omega} \cap \Omega$, we have

$$
\left|\pi_{t+1, \Omega^{*}}(g)(w)-\pi_{t+1, \Omega^{*}}(g)(\tilde{w})\right| \leq \varepsilon
$$


whenever $\left.\mid S_{t+1}(w)-S_{t+1}(\tilde{w})\right) \mid<\delta$. Note that $\Delta S_{t+1}(v)-\Delta S_{t+1}\left(v^{\prime}\right)$ is orthogonal to $H_{t+1}(\omega)$ and

$$
\begin{aligned}
& \varepsilon+\pi_{t, \Omega^{*}}(g)(g)(v)+\left(H_{t+1}(\omega)+\frac{\xi_{t+1, \Sigma_{t}^{\omega} \cap \Omega}(v)}{\delta / \varepsilon}\right) \\
& \times\left(\Delta S_{t+1}\left(v^{\prime}\right)+\Delta S_{t+1}(v)-\Delta S_{t+1}\left(v^{\prime}\right)\right) \\
& \geq \varepsilon+\pi_{t+1, \Omega^{*}}(g)\left(v^{\prime}\right)+\frac{S_{t+1}(v)-S_{t+1}\left(v^{\prime}\right)}{\delta / \varepsilon} \xi_{t+1, \Sigma_{t}^{\omega} \cap \Omega}(v) \\
& \geq \pi_{t+1, \Omega^{*}}(g)(v) .
\end{aligned}
$$

Next we use the assumption that $A_{t}^{\omega}$ is bounded and has no points of convergence in $\operatorname{span}\left(\Delta S_{t+1}\left(\Sigma_{t}^{\omega} \cap \Omega^{*}\right)\right)$ outside the set $\Delta S_{t+1}\left(\Sigma_{t}^{\omega} \cap \Omega^{*}\right)$. In particular, the continuous functions $\pi_{t+1, \Omega^{*}}(g)$ and $H_{t+1} \Delta S_{t+1}$ are bounded on $A_{t}^{\omega}$. There exists $\delta>0$ such that for all $v \in \Delta S_{t+1}\left(\Sigma_{t}^{\omega} \cap \Omega^{*}\right), \tilde{v} \in A_{t}^{\omega}$ with $|v-\tilde{v}|<\delta$, we still have

$$
\varepsilon+\pi_{t, \Omega^{*}}(g)(\omega)+H_{t+1}(\omega) \Delta S_{t+1}(\tilde{v}) \geq \pi_{t+1, \Omega^{*}}(g)(\tilde{v}) .
$$

By assumption, there exists $\tilde{\delta}>0$ such that $\operatorname{dist}\left(\tilde{v}, \operatorname{span}\left(\Delta S_{t+1}\left(\Sigma_{t}^{\omega} \cap \Omega^{*}\right)\right)\right)>\tilde{\delta}$ for all $\tilde{v} \in A_{t}^{\omega}$ with $\operatorname{dist}\left(\tilde{v}, \Delta S_{t+1}\left(\Sigma_{t}^{\omega} \cap \Omega^{*}\right)\right)>\delta$. Define

$$
\pi_{\max }=\sup _{v \in A_{t}^{\omega}} \pi_{t+1, \Omega^{*}}(g)(v)<\infty
$$

and $C=\inf _{v \in A_{t}^{\omega}} H_{t+1}(\omega) \Delta S_{t+1}(v)+\pi_{t, \Omega^{*}}(g)(\omega)>-\infty$. Now we note that

$$
\begin{aligned}
& \varepsilon+\pi_{t+1, \Omega^{*}}(g)(\tilde{v})+H_{t+1}(\tilde{v}) \Delta S_{t+1}(\tilde{v})+\frac{\left|\pi_{\max }\right|+|C|}{\tilde{\delta}} \xi_{t+1, \Sigma_{t}^{\omega} \cap \Omega}(v) \Delta S_{t+1}(\tilde{v}) \\
& \geq \pi_{t+1, \Omega^{*}}(g)(\tilde{v})
\end{aligned}
$$

for all $\tilde{v} \in A_{t}^{\omega}$. This concludes the proof.

\subsection{Notions of arbitrage: an overview of robust financial modelling}

One of the most important underlying concepts in financial mathematics is the absence of arbitrage. In the literature on robust pricing and hedging, many notions of arbitrage have been proposed to date. The aim of this section is to provide a comprehensive list of these different notions in a unified manner to facilitate their direct comparison and to discuss their relative dependencies. This could potentially offer an important point of reference for researchers in the field. To complement the picture, we establish some new technical results.

In this section, we work with a more general set $\Phi=\left\{\phi_{\lambda}: \lambda \in \Lambda\right\}$ of options for static trading; in particular, $\Phi$ need not be finite. However, we only allow trading positions $h$ which hold finitely many of these assets; so $h \in c_{00}(\Lambda)$, the space of sequences of reals indexed by $\Lambda$ with only finitely many non-zero elements. As before, $h \in c_{00}(\Lambda)$ generates the payoff $h \cdot \Phi=\sum_{\lambda \in \Lambda} h_{\lambda} \phi_{\lambda}$ at time $T$. We also adapt the 
notation for admissible trading strategies to be $\mathcal{A}_{\Phi}(\mathbb{F}):=c_{00}(\Lambda) \times \mathcal{H}(\mathbb{F})$. The following complements Definitions 2.3 and 2.6, where the notions of $\mathbf{1 p A}(\Omega), \mathbf{S A}(\Omega)$, $\mathbf{A}(\mathfrak{P}), \operatorname{Int} \mathbf{A}(\mathfrak{P})$ were introduced.

Definition 3.11 Fix a filtration $\mathbb{F}$ and sets $\mathfrak{P} \subseteq \mathcal{P}(X)$ and $\Omega \subseteq X$.

- OA $(\Omega)$ : An open arbitrage (see Riedel [34]) is a strategy $(h, H) \in \mathcal{A}_{\Phi}(\mathbb{F})$ such that $h \cdot \Phi+H \cdot S_{T} \geq 0$ on $\Omega$, with strict inequality for some open subset of $\Omega$.

- USA $(\Omega)$ : A uniformly strong arbitrage (see Davis and Hobson [23]) is a strategy $(h, H) \in \mathcal{A}_{\Phi}(\mathbb{F})$ such that $h \cdot \Phi+H \bullet S_{T} \geq \varepsilon$ on $\Omega$ for some $\varepsilon>0$.

- CA(P): A classical arbitrage in $\mathfrak{P}$ (see Davis and Hobson [23]) is a family of strategies $\left(h^{\mathbb{P}}, H^{\mathbb{P}}\right)_{\mathbb{P} \in \mathfrak{P}}$ such that $\left(h^{\mathbb{P}}, H^{\mathbb{P}}\right)$ is a $\mathbb{P}$-arbitrage for all $\mathbb{P} \in \mathfrak{P}$.

- WA(P): A weak arbitrage (see Blanchard and Carassus [10]) is a strategy $(h, H) \in \mathcal{A}_{\Phi}(\mathbb{F})$ which is a $\mathbb{P}$-arbitrage for some $\mathbb{P} \in \mathfrak{P}$.

- IntA(P): An interior arbitrage (see Bayraktar et al. [7]) is a sequence of strategies $\left(h^{n}, H^{n}\right) \in \mathcal{A}_{\Phi}(\mathbb{F})$ such that $\left(h^{n}, H^{n}\right)$ is a $\mathfrak{P}$-quasi-sure arbitrage relative to option payoffs given by $\Phi+\operatorname{sign}\left(h^{n}\right) / n$ for all $n$ large enough.

- WFLVR $(\Omega)$ : A weak free lunch with vanishing risk (see Cox and Obłój [21], Cox et al. [20]) is a sequence of strategies $\left(h^{n}, H^{n}\right) \in \mathcal{A}_{\Phi}(\mathbb{F})$ such that there exist a constant $c \geq 0$ and $(h, H) \in \mathcal{A}_{\Phi}(\mathbb{F})$ with $h^{n} \cdot \Phi+H^{n} \bullet S_{T} \geq h \cdot \Phi+H \bullet S_{T}-c$ on $\Omega$ for all $n \in \mathbb{N}$ and

$$
\lim _{n \rightarrow \infty}\left(h^{n} \cdot \Phi+H^{n} \cdot S_{T}\right)>0 \quad \text { on } \Omega \text {. }
$$

$-\operatorname{loc} \mathbf{A}\left(\mathfrak{P}_{t}(\omega)\right): \mathbf{A}(t, \omega)$-local $\mathfrak{P}$-quasi-sure arbitrage (see Bartl [3]) is a strategy $H \in \mathbb{R}^{d}$ such that $H \Delta S_{t+1}(\omega) \geq 0 \mathfrak{P}_{t}(\omega)$-q.s. (where $t \in\{0, \ldots, T-1\}$ and $\omega \in X$ ) and there exists $\mathbb{P} \in \mathfrak{P}_{t}(\omega)$ such that $\mathbb{P}\left[H \Delta S_{t+1}>0\right]>0$.

Lemma 3.12 The following relations hold:

1) $\mathbf{U S A}(\Omega) \Rightarrow \mathbf{S A}(\Omega) \Rightarrow \mathbf{O A}(\Omega) \Rightarrow \mathbf{1 p A}(\Omega)$.

2) $\mathbf{S A}(\Omega) \Rightarrow \mathbf{W F L V R}(\Omega)$.

3) $\mathbf{A}(\mathfrak{P}) \Rightarrow \mathbf{A}(\mathbb{P})$ for some $\mathbb{P} \in \mathfrak{P} \Leftrightarrow \mathbf{W A}(\mathfrak{P})$.

4) $\mathbf{W A}(\mathfrak{P}) \Leftarrow \mathbf{C A}(\mathfrak{P}) \Leftrightarrow \mathbf{A}(\mathbb{P})$ for all $\mathbb{P} \in \mathfrak{P}$.

5) $\mathbf{A}(\mathfrak{P}) \Rightarrow \operatorname{IntA}(\mathfrak{P})$.

6) If $\Phi=0, \mathbf{A}(\mathfrak{P})$ is equivalent to $\mathbb{P}\left[\bigcup_{t=0}^{T-1}\left\{\omega \in X_{t}: \operatorname{loc} \mathbf{A}\left(\mathfrak{P}_{t}(\omega)\right.\right.\right.$ holds $\left.\}\right]>0$ for some $\mathbb{P} \in \mathfrak{P}$.

Proof 1)-4) are immediate. 6) follows from Bouchard and Nutz [12, Lemma 4.6]. For a strategy $(h, H) \in \mathcal{A}_{\Phi}(\mathbb{F})$ satisfying

$$
h \cdot \Phi+H \cdot S_{T} \geq 0 \quad \mathfrak{P} \text {-q.s., }
$$

we have for any $\varepsilon>0$ that

$$
h \cdot(\Phi+\operatorname{sign}(h) \varepsilon)+H \cdot S_{T}=h \cdot \Phi+H \bullet S_{T}+|h|_{1} \varepsilon \geq|h|_{1} \varepsilon \geq 0,
$$


where $|h|_{1}=\sum_{\lambda \in \Lambda}\left|h_{\lambda}\right|$. The absence of $\operatorname{IntA}(\mathfrak{P})$ implies that there exists $\varepsilon>0$ such that for any strategy as above, we have

$$
h \cdot \Phi+H \cdot S_{T}=-|h|_{1} \varepsilon \quad \mathfrak{P} \text {-q.s., }
$$

so that $h=0$ and the absence of $\mathbf{A}(\mathfrak{P})$ follows and, in conclusion, 5) holds.

USA $(X)$ was first discussed in Davis and Hobson [23]; see also Cox and Obłój [21] and Cox et al. [20] for a definition of $\operatorname{USA}(\Omega)$ and $\operatorname{WFLVR}(\Omega)$ where $\Omega \subseteq X$. Note that if we take $(h, H)=(0,0)$ in the definition of $\operatorname{WFLVR}(\Omega)$ and replace the pathwise inequalities by their $\mathbb{P}$-a.s. counterparts for some fixed $\mathbb{P} \in \mathcal{P}(X)$, we recover a discrete version of the NFLVR condition of Delbaen and Schachermayer [24].

$\mathbf{S A}\left(\left(\mathbb{R}_{+}^{d}\right)^{T}\right)$ was used in Acciaio et al. [1] in the canonical setup. We refer to Burzoni et al. [13, Theorem 3] for a general FTAP connecting the notion of strong and uniformly strong arbitrage under the condition that there exists in the market an option with a strictly convex superlinear payoff. See also Bartl et al. [4] for an equivalence result under marginal constraints. Below we discuss the connection between $\mathbf{S A}(\Omega)$ and $\mathbf{U S A}(\Omega)$ without the above assumptions.

$\mathbf{A}(\mathcal{S})$ is a unifying concept since $1 \mathbf{p A}(\Omega), \mathbf{O A}(\Omega), \mathbf{S A}(\Omega), \mathbf{U S A}(\Omega)$ and $\mathbf{A}(\mathfrak{P})$ can all be seen as special cases of $\mathbf{A}(\mathcal{S})$; see Burzoni et al. [14, Sect. 4.6] for a detailed discussion. It was first defined in [14] in a pathwise setting; see in particular the pathwise fundamental theorem of asset pricing in [14, Theorem 2 and Sect. 4]. This extends the results obtained in Riedel [34] who introduced $1 \mathbf{p A}(\Omega)$ and $\mathbf{O A}(\Omega)$. $\mathbf{O A}(\Omega)$ is furthermore defined in the setup of Dolinsky and Soner [26].

$\mathbf{A}(\mathfrak{P})$ was introduced in the quasi-sure setting of Bouchard and Nutz [12] who prove a quasi-sure fundamental theorem of asset pricing and superhedging theorem. From Lemma 3.12 above, we see that the crucial distinction between $\mathbf{C A}(\mathfrak{P})$ and $\mathbf{A}(\mathfrak{P})$ is the aggregation of arbitrage strategies, which poses a fundamental technical difficulty overcome in Bouchard and Nutz [12] by the specific (APS) structure of $\mathfrak{P}$. We also note that $\mathbf{C A}(\mathfrak{P})$ was actually referred to as weak arbitrage in Davis and Hobson [23].

The notion of interior arbitrage $\mathbf{I n t A}(\mathfrak{P})$ was introduced, and called a robust arbitrage, by Bayraktar et al. [7] in the context of transaction costs. The absence of $\operatorname{IntA}(\mathfrak{P})$ is equivalent to the absence of $\mathbf{A}(\mathfrak{P})$ not only at the current prices of statically traded options $\Phi$, but also under all sufficiently small perturbations of their prices. This notion was also used in Hou and Obłój [28, Assumption 3.1]. It is equivalent to saying that the prices of the options $\Phi$ are strictly inside the region of their $\mathfrak{P}$-q.s. no-arbitrage prices, thus avoiding the delicate issue of boundary classification. In general, IntA $(\mathfrak{P})$ does not imply $\mathbf{A}(\mathfrak{P})$. To see this, take $\Phi=\left\{\left(S_{T}-K\right)^{+}\right\}$for some $K>S_{0}$ and $\emptyset \neq \mathfrak{P} \subseteq\left\{\mathbb{P} \in \mathcal{P}(X): \mathbb{P}\left[S_{T} \leq K\right]=1\right\}$. Then there is no $\mathfrak{P}$-q.s. arbitrage, while for every $\varepsilon>0$ we have $\left(S_{T}-K\right)^{+}+\varepsilon \geq \varepsilon>0$ and thus $\operatorname{IntA}(\mathfrak{P})$ holds.

We now take a closer look at the notions $\mathbf{S A}(\Omega)$ and $\mathbf{U S A}(\Omega)$ and establish their equivalence in specific market setups. Clearly, every uniformly strong arbitrage is a strong arbitrage. In general, the opposite assertion is not true. Take for example $d=1, S_{0}=1, S_{1}(\omega)=\omega, \Omega=(1,2]$; then every $H_{1}>0$ is a strong arbitrage, but 
there exist no uniformly strong arbitrages. This simple example can be generalised: Any one-period market in the canonical setting with $S_{0}=1$ and an open convex set $\Omega$ such that $\{1\} \cap \Omega=\emptyset$ and $1 \in \bar{\Omega}$ admits a strong arbitrage, but exhibits no uniformly strong arbitrages. On the level of superhedging prices, a uniformly strong arbitrage on $\Omega$ corresponds to $\pi_{\Omega}(0)=-\infty$. For a financial market which exhibits a strong arbitrage but no uniformly strong arbitrages, the pricing-hedging duality cannot hold (as there are no martingale measures supported on $\Omega$ ), but $\pi_{\Omega}(0)=0$. In conclusion, the difference between uniformly strong arbitrage and strong arbitrage can be seen as a property describing the boundary of the prediction set $\Omega$ and thus manifests itself in the boundary behaviour of the superhedging functional

$$
S_{0} \mapsto \inf \left\{x \in \mathbb{R}: \exists H \in \mathbb{R}^{d} \text { such that } x+H\left(S_{1}-S_{0}\right) \geq 0 \text { on } \Omega\right\} \text {. }
$$

As this is an upper semicontinuous function, it takes the value zero on the boundary of $\Omega$, while its lower semicontinuous version takes the value $-\infty$. Nevertheless, the two notions agree in specific cases which we now explore.

We assume the canonical setting $S_{0}(\omega)=s_{0}, S_{t}(\omega)=\omega_{t}, X_{1}=\mathbb{R}_{+}^{d}$ and set $\mathcal{F}_{t}=\mathcal{F}_{t}^{0}$ for all $t=0,1, \ldots, T$. For the rest of this section, we allow countably many statically traded options, $\Lambda=\mathbb{N}$, but only of European type,

$$
\Phi=\left\{\phi_{n}=\phi_{n}\left(S_{T}\right): n \in \mathbb{N}\right\}
$$

with real-valued continuous payoff functions and a common maturity $T$. We write $c_{00}=c_{00}(\mathbb{N})$ for simplicity. We fix a closed subset $\Omega \subseteq\left(\mathbb{R}_{+}^{d}\right)^{T}$ and recall that martingale measures on $\Omega$ calibrated to $\Phi$ are denoted by $\mathcal{M}_{\Omega, \Phi}(\mathbb{F})$. We define $|S(\omega)|_{1}:=\sum_{t=1}^{T} \sum_{k=1}^{d}\left|S_{t}^{k}(\omega)\right|$ and denote by $C_{|S|_{1}}^{b}(\Omega)$ the space of real-valued continuous functions $f: \Omega \mapsto \mathbb{R}$ such that

$$
\sup _{\omega \in \Omega} \frac{|f(\omega)|}{|S(\omega)|_{1} \vee 1}<\infty .
$$

Finally, we define the calibrated supermartingale measures as

$$
\begin{aligned}
\mathcal{S} \mathcal{M}_{\Omega, \Phi}(\mathbb{F}):=\{\mathbb{Q} \in \mathcal{P}(\Omega): & \mathbb{E}_{\mathbb{Q}}\left[\phi_{n}\right] \leq 0, \forall n \in \mathbb{N}, \\
& \left.\mathbb{E}_{\mathbb{Q}}\left[S_{t} \mid \mathcal{F}_{t-1}\right] \leq S_{t-1} \text { a.s., } \forall t \leq T\right\} .
\end{aligned}
$$

In the above setup, we can prove:

Theorem 3.13 The following hold:

1) $\boldsymbol{S A}(\Omega) \Leftrightarrow \boldsymbol{U S A}(\Omega)$.

2) Assume $\phi_{n} \in C_{|S|_{1}}^{b}(\Omega)$, no short-selling in any of the assets and

$$
\lim _{\left|S_{T}\right|_{1} \rightarrow \infty} \frac{\left(\phi_{n}\left(S_{T}\right)\right)^{-}}{\left|S_{T}\right|_{1}}=0
$$

for all $n \in \mathbb{N}$. Then $\boldsymbol{S A}(\Omega) \Leftrightarrow \boldsymbol{U S A}(\Omega) \Leftrightarrow \operatorname{WFLVR}(\Omega) \Leftrightarrow \mathcal{S M}_{\Omega, \Phi}(\mathbb{F})=\emptyset$. 
3) As in 2), assume that $\phi_{n} \in C_{|S|_{1}}^{b}(\Omega)$. Furthermore, assume that for every sequence $\left(\omega_{n}\right)_{n \in \mathbb{N}}$ with $\lim _{n \rightarrow \infty}\left|S\left(\omega_{n}\right)\right|_{1}=\infty$, there exist a sequence $\left(h^{k}, H^{k}\right)_{k \in \mathbb{N}}$ of trading strategies, a constant $C>0$ and a sequence $\left(p^{k}\right)_{k \in \mathbb{N}}$ such that

$-\lim _{k \rightarrow \infty} \lim _{n \rightarrow \infty} \frac{\left(h^{k} \cdot \Phi+H^{k} \bullet S_{T}\right)\left(\omega_{n}\right)}{\left|S\left(\omega_{n}\right)\right|_{1} \vee 1}>0$,

- $\left|h^{k} \cdot \Phi+H^{k} \cdot S_{T}\right| \leq C\left(|S|_{1} \vee 1\right)$ on $\Omega$ for all $k \in \mathbb{N}$,

- $\lim _{k \rightarrow \infty}\left(h^{k} \cdot \Phi+H^{k} \cdot S_{T}\right)(\omega)=-\lim _{k \rightarrow \infty} p^{k}$ for all $\omega \in \Omega$.

Then $\boldsymbol{S A}(\Omega) \Leftrightarrow \boldsymbol{U S A}(\Omega) \Rightarrow \boldsymbol{W F L V R}(\Omega) \Leftrightarrow \mathcal{M}_{\Omega, \Phi}(\mathbb{F})=\emptyset$, but in general $\operatorname{WFLVR}(\Omega)$ does not imply $\operatorname{SA}(\Omega)$.

Remark 3.14 The above theorem can be seen as a unification of Acciaio et al. [1, Theorem 1.3], Cox and Obłój [21, Proposition 2.2] and Bartl et al. [4, Corollary 4.6]. We also refer to Burzoni et al. [13, Theorem 3], who extended Acciaio et al. [1, Theorem 1.3] under the assumption $\Omega=\Omega^{*}$, and to Burzoni et al. [16, Theorem C.5] for a general discussion in the case $\Phi=0$. In contrast to the work of Acciaio et al. [1], we do not assume the existence of a convex superlinear payoff $g$, which might be artificial in some settings, but we explicitly enforce tightness of martingale measures through the WFLVR $(\Omega)$ condition.

Remark 3.15 1) The case $|\Phi|<\infty$ is covered in Bouchard and Nutz [12], Burzoni et al. [13], while the case $|\Phi|=\infty$ is not. The basic idea in both works is to inductively construct a martingale measure calibrated to a finite number of options.

2) Contrary to the case $|\Phi|<\infty$ (see Burzoni et al. [13, proof of Theorem 1]), the set $\mathcal{M}_{\Omega, \Phi}(\mathbb{F})$ might not necessarily contain any finitely supported martingale measures.

3) An example showing that $\operatorname{WFLVR}(\Omega)$ does not imply $\mathbf{S A}(\Omega)$ is given in Cox and Obłój [21, Proposition 2.2].

4) A special but important case of 3 ) in Theorem 3.13 is $T=1, d=1$ and call options $\phi_{n}$ with payoff $\phi_{n}\left(S_{1}\right)=\left(S_{1}-n\right)^{+}-p^{n}$, where $p^{n} \geq 0$. In this case, we can set $H^{k}=0, h^{k}=e_{k}$ for all $k \in \mathbb{N}$, where $e_{k}$ is the $k$ th unit vector in $c_{00}$, and note that

$$
\begin{aligned}
& \lim _{k \rightarrow \infty} \lim _{n \rightarrow \infty} \frac{\left(h^{k} \cdot \Phi+H^{k}\left(S_{1}-S_{0}\right)\right)\left(\omega_{n}\right)}{\left|S\left(\omega_{n}\right)\right|_{1} \vee 1} \\
& =\lim _{k \rightarrow \infty} \lim _{n \rightarrow \infty} \frac{\left(S_{1}\left(\omega_{n}\right)-k\right)^{+}-p^{k}}{\left|S\left(\omega_{n}\right)\right|_{1}}=1>0
\end{aligned}
$$

and

$$
\lim _{k \rightarrow \infty}\left(S_{1}(\omega)-k\right)^{+}-p^{k}=-\lim _{k \rightarrow \infty} p^{k}
$$

In particular, all three conditions in 3) are satisfied.

Proof of Theorem 3.13 For simplicity of exposition, we only give the proof for $T=1$. This conveys the important ideas, while the multi-period case extends these via a dynamic programming approach and can found in Wiesel [42, Sect. 2.4.3]. 
Regarding 1$)$, clearly $\mathbf{U S A}(\Omega) \Rightarrow \mathbf{S A}(\Omega)$; so we show $\mathbf{S A}(\Omega) \Rightarrow \mathbf{U S A}(\Omega)$. Let $(h, H) \in \mathbb{R}^{k} \times \mathbb{R}^{d}$ be a strong arbitrage. We show that it is actually a uniformly strong arbitrage. For $x \in \mathbb{R}_{+}^{d}$, we denote by $|x|_{1}:=\sum_{i=1}^{d} x^{i}$ the $\ell_{1}$-norm of $x$ and define the compact set

$$
K=\left[0, s_{0}^{1}+2\left|s_{0}\right|_{1}\right] \times\left[0, s_{0}^{2}+2\left|s_{0}\right|_{1}\right] \times \cdots \times\left[0, s_{0}^{d}+2\left|s_{0}\right|_{1}\right] .
$$

Then, as $S_{1} \mapsto h \cdot \Phi\left(S_{1}\right)+H\left(S_{1}-S_{0}\right)$ is continuous and positive on the compact set $\Omega \cap K$, there exists $\varepsilon>0$ such that

$$
h \cdot \Phi\left(S_{1}\right)+H \cdot\left(S_{1}-S_{0}\right) \geq \varepsilon \quad \text { on } K \cap \Omega \text {. }
$$

Scaling $(h, H)$ suitably, we can without loss of generality take $\varepsilon=2\left|s_{0}\right|_{1}$. Let $\mathbf{e}=(1, \ldots, 1)$ be the row unit vector in $\mathbb{R}^{d}$. Then

$$
h \cdot \Phi\left(S_{1}\right)+(H+\mathbf{e}) \cdot\left(S_{1}-S_{0}\right) \geq 2\left|s_{0}\right|_{1}-\left|s_{0}\right|_{1}=\left|s_{0}\right|_{1} \quad \text { on } K \cap \Omega .
$$

Furthermore, on $\Omega \backslash K$, we have

$$
h \cdot \Phi\left(S_{1}\right)+(H+\mathbf{e}) \cdot\left(S_{1}-S_{0}\right) \geq \mathbf{e} \cdot\left(S_{1}-S_{0}\right) \geq 2\left|s_{0}\right|_{1}-\left|s_{0}\right|_{1}=\left|s_{0}\right|_{1} .
$$

Now we show 2). Clearly, the relation $\operatorname{USA}(\Omega) \Rightarrow \operatorname{WFLVR}(\Omega)$ holds and by 1$)$ also $\mathbf{S A}(\Omega) \Leftrightarrow \operatorname{USA}(\Omega)$. Further, $\operatorname{WFLVR}(\Omega)$ readily implies $\mathcal{S} \mathcal{M}_{\Omega, \Phi}(\mathbb{F})=\emptyset$ since otherwise if $\mathbb{Q} \in \mathcal{S} \mathcal{M}_{\Omega, \Phi}(\mathbb{F})$, then by Fatou's lemma,

$$
\begin{aligned}
0 & \geq \liminf _{n \rightarrow \infty} \mathbb{E}_{\mathbb{Q}}\left[h^{n} \cdot \Phi+H^{n}\left(S_{1}-S_{0}\right)\right] \\
& \geq \mathbb{E}_{\mathbb{Q}}\left[\liminf _{n \rightarrow \infty}\left(h^{n} \cdot \Phi+H^{n}\left(S_{1}-S_{0}\right)\right)\right]>0,
\end{aligned}
$$

which is a contradiction. Next we show $\operatorname{NUSA}(\Omega) \Rightarrow\left(\mathcal{S M}_{\Omega, \Phi}(\mathbb{F}) \neq \emptyset\right)$ following closely the argument in Acciaio et al. [1, proof of Proposition 2.3 and Theorem 1.3]. We denote by $c_{00}^{+}$the subset of all nonnegative sequences in $c_{00}$. We define the set

$$
K:=\left\{h \cdot \Phi\left(S_{1}\right)+H\left(S_{1}-S_{0}\right):(h, H) \in c_{00}^{+} \times \mathbb{R}_{+}^{d}\right\} \subseteq C_{|S|_{1}}^{b}(\Omega) .
$$

Note that $K$ is convex and nonempty. Furthermore, denote the positive cone of $C_{|S|_{1}}^{b}(\Omega)$ by

$$
C_{++}(\Omega)=\left\{f \in C_{|S|_{1}}^{b}(\Omega): \inf _{\omega \in \Omega} \frac{f(\omega)}{|S(\omega)|_{1} \vee 1}>0\right\} .
$$

By NUSA $(\Omega)$, we have $K \cap C_{++}(\Omega)=\emptyset$. An application of the Hahn-Banach theorem yields the existence of a positive measure $\mu$ such that

$$
\begin{array}{ll}
\int_{\Omega} \frac{f}{|S|_{1} \vee 1} d \mu>0 & \text { for all } f \in C_{++}(\Omega), \\
\int_{\Omega} \frac{f}{|S|_{1} \vee 1} d \mu \leq 0 & \text { for all } f \in K .
\end{array}
$$


We write $\mu=\mu^{r}+\mu^{s}$ for the decomposition of $\mu$ into its regular and singular parts with respect to the Stone-Čech compactification of $\left(\mathbb{R}_{+}^{d}\right)^{T}$. We now aim to show that the normalised measure $\mathbb{Q}$ given by

$$
d \mathbb{Q}:=\frac{1}{|S|_{1} \vee 1}\left(\int \frac{1}{|S|_{1} \vee 1} d \mu^{r}\right)^{-1} d \mu^{r}
$$

is an element of $\mathcal{S} \mathcal{M}_{\Omega, \Phi}$. First, observe that $\mu^{r}$ is non-zero. Indeed, if $\mu=\mu^{s}$, then

$$
\int_{\Omega} \frac{\mathbf{e}\left(S_{1}-S_{0}\right)}{|S|_{1} \vee 1} d \mu=\int_{\Omega} \frac{|S|_{1}-\left|S_{0}\right|_{1}}{|S|_{1} \vee 1} d \mu^{s}=\int_{\Omega} 1 d \mu^{s}>0
$$

as $\mu$ is positive and $\Omega \subseteq\left(\mathbb{R}_{+}^{d}\right)^{T}$, which is a contradiction. As $\int_{\Omega} \frac{\left(\phi_{n}\left(S_{1}\right)\right)^{-}}{|S|_{1} \vee 1} d \mu^{s}=0$, we conclude that

$$
\int_{\Omega} \frac{\phi_{n}\left(S_{1}\right)}{|S|_{1} \vee 1} d \mu^{r} \leq \int_{\Omega} \frac{\phi_{n}\left(S_{1}\right)}{|S|_{1} \vee 1} d \mu \leq 0 \quad \text { for all } n \in \mathbb{N} .
$$

Analogously, we conclude that

$$
\int_{\Omega} \frac{S_{1}-S_{0}}{|S|_{1} \vee 1} d \mu^{r} \leq 0
$$

and thus $\mathbf{N U S A}(\Omega) \Rightarrow \mathcal{S} \mathcal{M}_{\Omega, \Phi} \neq \emptyset$ follows.

Finally, we show 3), following the same construction as in 2). In particular, redefining

$$
K:=\left\{h \cdot \Phi\left(S_{1}\right)+H\left(S_{1}-S_{0}\right):(h, H) \in c_{00} \times \mathbb{R}^{d}\right\} \subseteq C_{|S|_{1}}^{b}(\Omega),
$$

we note that again by $\operatorname{NUSA}(\Omega)$, we have $K \cap C_{++}(\Omega)=\emptyset$. Thus all that is left to show is $\mu^{s}=0$. Let us assume towards a contradiction $\mu^{s} \neq 0$ and take $\left(h^{k}, H^{k}\right)_{k \in \mathbb{N}}$ such that

$$
\lim _{k \rightarrow \infty} \int_{\Omega} \frac{h^{k} \cdot \Phi\left(S_{1}\right)+H^{k}\left(S_{1}-S_{0}\right)}{|S|_{1} \vee 1} d \mu^{s}>0 .
$$

Then by symmetry of $K$ and the same reasoning as in 2), we have

$$
\int_{\Omega} \frac{h^{k} \cdot \Phi\left(S_{1}\right)+H^{k}\left(S_{1}-S_{0}\right)}{|S|_{1} \vee 1} d \mu=0 \quad \text { for all } k \in \mathbb{N} .
$$

Using (3.1) and (3.2) gives

$$
\begin{aligned}
& \lim _{k \rightarrow \infty} \int_{\Omega} \frac{h^{k} \cdot \Phi\left(S_{1}\right)+H^{k}\left(S_{1}-S_{0}\right)}{|S|_{1} \vee 1} d \mu^{r} \\
& =-\lim _{k \rightarrow \infty} \int_{\Omega} \frac{h^{k} \cdot \Phi\left(S_{1}\right)+H^{k}\left(S_{1}-S_{0}\right)}{|S|_{1} \vee 1} d \mu^{s}<0 .
\end{aligned}
$$


Note that for a sequence $\left(p^{k}\right)_{n \in \mathbb{N}}$ with

$$
\lim _{k \rightarrow \infty} h^{k} \cdot \Phi\left(S_{1}\right)+H^{k}\left(S_{1}-S_{0}\right)=-\lim _{k \rightarrow \infty} p^{k}
$$

for all $\omega \in \Omega$, we need to have by no $\operatorname{WFLVR}(\Omega)$ that $\lim _{k \rightarrow \infty} p^{k}=0$; so the lefthand side of (3.3) is equal to zero, which is a contradiction.

\section{Technical results and proofs}

\subsection{Proof of Theorems 2.5 and 2.7}

We start with the following technical observation.

Proposition 4.1 Let $\Omega$ be analytic. Then the FTAP of Bouchard and Nutz [12] implies

$$
\operatorname{N1pA}\left(\Omega, \mathbb{F}^{\mathcal{U}}\right) \Longleftrightarrow \Omega=\Omega_{\Phi}^{*}
$$

Remark 4.2 The key argument is to show that for any analytic set $\Omega$, the corresponding set of finitely supported measures $\mathcal{P}^{f}\left(\operatorname{proj}_{t+1}\left(\Omega \cap \Sigma_{t}^{\omega}\right)\right)$ for every time period $t=1, \ldots, T$ has analytic graph. This allows us to use results from [12].

Proof of Proposition 4.1 Set $\hat{\mathfrak{P}}:=\mathcal{P}^{f}(\Omega)$. As just noted, to apply the FTAP of [12], we only need to show that $\hat{\mathfrak{P}}_{t}(\omega):=\mathcal{P}^{f}\left(\operatorname{proj}_{t+1}\left(\Omega \cap \Sigma_{t}^{\omega}\right)\right)$ has analytic graph. We therefore fix $n \in \mathbb{N}$ and consider the Borel-measurable function

$$
\begin{aligned}
\Sigma: X^{n} & \rightarrow X^{n} \times\left(\mathbb{R}_{+}^{d}\right)^{(t+1) n} \\
\left(\omega^{1}, \ldots, \omega^{n}\right) & \mapsto\left(\omega^{1}, \ldots, \omega^{n}, S_{0: t}\left(\omega^{1}\right), \ldots, S_{0: t}\left(\omega^{n}\right)\right)
\end{aligned}
$$

and note that the image $\Sigma\left(\Omega^{n}\right)$ is analytic, since $\Omega$ is analytic and the image of an analytic set under a Borel-measurable map as well as the Cartesian product of analytic sets is analytic (see [8, Propositions 7.38 and 7.40]). Next we consider the continuous function

$$
\begin{aligned}
F: X^{n} \times\left(\mathbb{R}_{+}^{d}\right)^{(t+1)} & \rightarrow X^{n} \times\left(\mathbb{R}_{+}^{d}\right)^{(t+1) n}, \\
\left(\omega^{1}, \ldots, \omega^{n}, x\right) & \mapsto\left(\omega^{1}, \ldots, \omega^{n}, x, \ldots, x\right) .
\end{aligned}
$$

Note that $F\left(X^{n} \times\left(\mathbb{R}_{+}^{d}\right)^{(t+1)}\right) \cap \Sigma\left(\Omega^{n}\right)$ is analytic, and as projections of analytic sets are analytic,

$$
A_{n}:=\left\{\left(\omega, \tilde{\omega}_{1}, \ldots, \tilde{\omega}_{n}\right): \omega \in \Omega, \tilde{\omega}_{i} \in \operatorname{proj}_{t+1}\left(\Omega \cap \Sigma_{t}^{\omega}\right), i=1, \ldots, n\right\}
$$


is analytic as well. Let $\Delta_{n} \subseteq \mathbb{R}^{n}$ denote the simplex. Since the functions

$$
\begin{aligned}
G: A_{n} \times \Delta_{n} & \rightarrow \Omega \times \mathcal{P}\left(X_{n}\right) \times \Delta_{n}, \\
\left(\omega, \tilde{\omega}_{1}, \ldots, \tilde{\omega}_{n}, \lambda_{1}, \ldots, \lambda_{n}\right) & \mapsto\left(\omega, \delta_{\tilde{\omega}_{1}}, \ldots, \delta_{\tilde{\omega}_{n}}, \lambda_{1}, \ldots, \lambda_{n}\right), \\
H: \Omega \times \mathcal{P}\left(X_{n}\right) \times \Delta_{n} & \rightarrow \Omega \times \mathcal{P}\left(X_{1}\right), \\
\left(\omega, \delta_{\tilde{\omega}_{1}}, \ldots, \delta_{\tilde{\omega}_{n}}, \lambda_{1}, \ldots, \lambda_{n}\right) & \mapsto\left(\omega, \sum_{i=1}^{n} \delta_{\tilde{\omega}_{i}} \lambda_{i}\right)
\end{aligned}
$$

are continuous, it follows that graph $\left(\hat{\mathfrak{P}}_{t}\right)=\bigcup_{n \in \mathbb{N}} H\left(G\left(A_{n} \times \Delta_{n}\right)\right)$ is analytic.

Take now $\omega \in \Omega$ and $\mathbb{P} \in \mathcal{P}^{f}(\Omega)$ such that $\mathbb{P}[\{\omega\}]>0$. By the FTAP of [12], there exists $\mathbb{Q} \in \mathcal{M}$ such that $\mathbb{Q} \ll \tilde{\mathbb{P}}$ for some $\tilde{\mathbb{P}} \in \mathcal{P}^{f}(\Omega), \mathbb{E}_{\mathbb{Q}}\left[\phi_{j}\right]=0$ for all $j=1, \ldots, k$ and $\mathbb{P} \ll \mathbb{Q}$. In particular, $\mathbb{Q} \in \mathcal{M}_{\Omega}^{f}$ and $\mathbb{Q}[\{\omega\}]>0$.

Finally, assume that $\Omega=\Omega_{\Phi}^{*}$ and fix $\mathbb{P} \in \hat{\mathfrak{P}}$ such that $\operatorname{supp}(\mathbb{P})=\left\{\omega_{1}, \ldots, \omega_{n}\right\}$ for some $n \in \mathbb{N}$. We can find $\mathbb{Q}_{1}, \ldots, \mathbb{Q}_{n} \in \mathcal{M}_{\Omega}^{f}$ such that $\mathbb{Q}_{i}\left[\left\{\omega_{i}\right\}\right]>0$ for $i=1, \ldots, n$. Then $\mathbb{Q}:=1 / n \sum_{i=1}^{n} \mathbb{Q}_{i} \in \mathcal{M}_{\Omega, \Phi}^{f}$ and $\mathbb{Q}\left[\left\{\omega_{i}\right\}\right]>0$ for $i=1, \ldots, n$, i.e., $\mathbb{P} \ll \mathbb{Q}$.

We now give a complete proof of the quasi-sure FTAP in [12] using results from [13]. We first look at the case $\Phi=0$ and start with an auxiliary lemma.

Lemma 4.3 Let $t \in\{1, \ldots, T\}$ and $\Omega \subseteq X_{t}$ be analytic. Then the conditional standard separator of Burzoni et al. [13] denoted by $\xi_{t, \Omega}$ is $\mathcal{F}_{t-1}^{\mathcal{U}}$-measurable.

Proof We shortly recall arguments from [13, proof of Lemma 1]. Let us define the multifunction

$$
\psi_{t, \Omega}: X \ni \omega \rightarrow\left\{\Delta S_{t}(\tilde{\omega}): \tilde{\omega} \in \Sigma_{t-1}^{\omega} \cap \Omega\right\} \subseteq \mathbb{R}^{d} .
$$

Then $\psi_{t, \Omega}$ is an $\mathcal{F}_{t-1}^{\mathcal{U}}$-measurable multifunction. Indeed, for $O \subseteq \mathbb{R}^{d}$ open, we have

$$
\left\{\omega \in X: \psi_{t, \Omega}(\omega) \cap O \neq \emptyset\right\}=S_{0: t-1}^{-1}\left(S_{0: t-1}\left(\left(\Delta S_{t}\right)^{-1}(O) \cap \Omega\right)\right) .
$$

As $\Delta S_{t}$ is Borel-measurable, $\left(\Delta S_{t}\right)^{-1}(O) \in \mathcal{F}_{t}^{0}$. Also as intersections, projections and preimages of analytic sets are analytic (see [8, Propositions 7.35 and 7.40]), we find that $\left\{\omega \in X: \psi_{t, \Omega}(\omega) \cap O \neq \emptyset\right\}$ is analytic and in particular $\mathcal{F}_{t-1}^{\mathcal{U}}$-measurable. Let $\mathbb{S}^{d}$ be the unit sphere in $\mathbb{R}^{d}$; then by preservation of measurability, also the multifunction

$$
\psi_{t, \Omega}^{*}(\omega):=\left\{H \in \mathbb{S}^{d}: H \cdot y \geq 0 \text { for all } y \in \psi_{t, \Omega}(\omega)\right\}
$$

is $\mathcal{F}_{t-1}^{\mathcal{U}}$-measurable and closed-valued. Let $\left(\xi_{t, \Omega}^{n}\right)_{n \in \mathbb{N}}$ be its $\mathcal{F}_{t-1}^{\mathcal{U}}$-measurable Castaing representation. The conditional standard separator is then defined as

$$
\xi_{t, \Omega}=\sum_{n=1}^{\infty} \frac{1}{2^{n}} \xi_{t, \Omega}^{n} .
$$


Remark 4.4 We recall that this separator has the property that it aggregates all onedimensional one-point arbitrages on $\Sigma_{t-1}^{\omega} \cap \Omega$ in the sense that

$$
\left\{\omega \in X: \xi(\omega) \cdot \Delta S_{t}(\omega)>0\right\} \subseteq\left\{\omega \in X: \xi_{t, \Omega}(\omega) \cdot \Delta S_{t}(\omega)>0\right\}
$$

for every measurable selector $\xi$ of $\psi_{t, \Omega}^{*}$.

Proof of Theorem 2.5 for $\Phi=0$ We start by proving the first part of Theorem 2.5, i.e., we are given a set of measures $\mathfrak{P}$ satisfying (APS) and we need to construct $\Omega=\Omega^{\mathfrak{P}}$ such that 1)-3) are equivalent. We define for $\omega \in X_{t-1}$ the set

$\tilde{\chi}_{\mathcal{F}_{t-1}^{0}}(\omega)$ as the intersection of all closed $A \subseteq \mathbb{R}^{d}$

$$
\text { with } \mathbb{P}\left[\Delta S_{t}(\omega, \cdot) \in A\right]=1, \forall \mathbb{P} \in \mathfrak{P}_{t-1}(\omega) \text {. }
$$

Then $\tilde{\chi}_{\mathcal{F}_{t-1}^{0}}$ is closed-valued and $\mathbb{P}\left[\Delta S_{t}(\omega, \cdot) \in \tilde{\chi}_{\mathcal{F}_{t-1}^{0}}(\omega)\right]=1$ for all $\mathbb{P} \in \mathfrak{P}_{t-1}(\omega)$ and all $\omega \in X_{t-1}$. Evidently,

$$
\begin{aligned}
\tilde{\chi}_{\mathcal{F}_{t-1}^{0}}(\omega)=\left\{x \in \mathbb{R}^{d}: \forall \varepsilon>0, \mathbb{P}\left[\Delta S_{t}(\omega, \cdot) \in B(x, \varepsilon)\right]>0\right. \\
\text { for some } \left.\mathbb{P} \in \mathfrak{P}_{t-1}(\omega)\right\} \\
=\frac{\bigcup_{\mathbb{P} \in \mathfrak{P}_{t-1}(\omega)} \operatorname{supp}\left(\mathbb{P} \circ\left(\Delta S_{t}(\omega, \cdot)\right)^{-1}\right) .}{}
\end{aligned}
$$

Also it follows from [12, Lemma 4.3] that $\tilde{\chi}_{\mathcal{F}_{t-1}^{0}}$ is analytically measurable. We quickly repeat their argument: let us define

$$
\ell: X_{t-1} \times \mathcal{P}\left(X_{1}\right) \rightarrow \mathcal{P}\left(\mathbb{R}^{d}\right), \quad \ell(\omega, \mathbb{P})=\mathbb{P} \circ\left(\Delta S_{t}(\omega, \cdot)\right)^{-1} .
$$

Then $\ell$ is Borel-measurable. Next we consider

$$
\begin{aligned}
\mathcal{R} & : X_{t-1} \rightarrow \mathcal{P}\left(\mathbb{R}^{d}\right), \\
\mathcal{R}(\omega) & :=\ell\left(\omega, \mathfrak{P}_{t-1}(\omega)\right)=\left\{\mathbb{P} \circ\left(\Delta S_{t}(\omega, \cdot)\right)^{-1}: \mathbb{P} \in \mathfrak{P}_{t-1}(\omega)\right\} .
\end{aligned}
$$

Since its graph is analytic, it follows that for $O \subseteq \mathbb{R}^{d}$ open,

$$
\begin{aligned}
\left\{\omega \in X_{t-1}: \tilde{\chi}_{\mathcal{F}_{t-1}^{0}}(\omega) \cap O \neq \emptyset\right\} & =\left\{\omega \in X_{t-1}: R[O]>0 \text { for some } R \in \mathcal{R}(\omega)\right\} \\
& =\operatorname{proj}_{X_{t-1}}\{(\omega, R) \in \operatorname{graph}(\mathcal{R}): R[O]>0\}
\end{aligned}
$$

is analytic as $R \mapsto R[O]$ is Borel.

We also note that for $\varepsilon>0$, the function $x \mapsto R\left[B_{\varepsilon}(x)\right]$ is continuous. Therefore $(x, R) \mapsto R\left[B_{\varepsilon}(x)\right]$ is Borel and

$$
\begin{aligned}
\operatorname{graph}\left(\tilde{\chi}_{\mathcal{F}_{t-1}^{0}}\right) & =\left\{(\omega, x) \in\left(X_{t-1} \times \mathbb{R}^{d}\right): x \in \tilde{\chi}_{\mathcal{F}_{t-1}^{0}}(\omega)\right\} \\
& =\bigcap_{\varepsilon \in \mathbb{Q}_{+}} \operatorname{proj}_{X_{t-1} \times \mathbb{R}^{d}}\left(\left\{(\omega, R, x) \in\left(\operatorname{graph}(\mathcal{R}) \times \mathbb{R}^{d}\right): R\left[B_{\varepsilon}(x)\right]>0\right\}\right)
\end{aligned}
$$


is analytic. Now we define

$$
U=\left\{\omega \in X_{t}: \Delta S_{t}(\omega) \in \tilde{\chi}_{\mathcal{F}_{t-1}^{0}}(\omega)\right\}
$$

Then

$$
U=\operatorname{proj}_{X_{t}}\left(\operatorname{graph}\left(\Delta S_{t}\right) \cap \operatorname{graph}\left(\tilde{\chi}_{\mathcal{F}_{t-1}^{0}}\right)\right)
$$

is analytic and by Fubini's theorem, $\mathbb{P}[U]=1$ holds for all $\mathbb{P} \in \mathfrak{P}$. We now set

$$
\Omega^{\mathfrak{P}}=\bigcap_{t=1}^{T}\left\{\omega \in X_{t}: \Delta S_{t}(\omega) \in \tilde{\chi}_{\mathcal{F}_{t-1}^{0}}(\omega)\right\},
$$

which is again analytic and $\mathbb{P}\left[\Omega^{\mathfrak{P}}\right]=1$ for all $\mathbb{P} \in \mathfrak{P}$.

Having defined $\Omega^{\mathfrak{P}}$, we can now begin to prove the equivalence of 1)-3). If 2) holds, then 3 ) follows immediately by a contradiction argument; so we now show the more involved implications 3$) \Rightarrow 1$ ) and 1) $\Rightarrow 2$ ). Let us start with the proof of $3) \Rightarrow 1$ ): we assume that there exists $\hat{\mathbb{P}} \in \mathfrak{P}$ such that $\hat{\mathbb{P}}\left[\Omega^{\mathfrak{P}} \backslash\left(\Omega^{\mathfrak{P}}\right)^{*}\right]>0$. We want to find $H \in \mathcal{H}\left(\mathbb{F}^{\mathcal{U}}\right)$ and $\tilde{\mathbb{P}} \in \mathfrak{P}$ such that $H \bullet S_{T} \geq 0 \mathfrak{P}$-q.s. and $\tilde{\mathbb{P}}\left[H \bullet S_{T}>0\right]>0$. For this, we take $t=T-1$ and assume that

$$
\hat{\mathbb{P}}\left[\left\{\omega \in \operatorname{proj}_{0: T-1}\left(\Omega^{\mathfrak{P}}\right): \text { there is a one-point arbitrage on } \Sigma_{T-1}^{\omega} \cap \Omega^{\mathfrak{P}}\right\}\right]>0 \text {. }
$$

Let us now fix

$$
\omega \in\left\{\operatorname{proj}_{0: T-1}\left(\Omega^{\mathfrak{P}}\right): \text { there is a one-point arbitrage on } \Sigma_{T-1}^{\omega} \cap \Omega^{\mathfrak{P}}\right\} \text {. }
$$

Denote by $\xi_{T, \Omega \mathfrak{P}}$ the $\mathcal{F}_{T-1}^{\mathcal{U}}$-measurable standard separator of Lemma 4.3. Now we recall the notation of the push-forward of $\mathbb{P}$ for each $\mathbb{P} \in \mathfrak{P}_{T-1}(\omega)$ given by

$$
\mathbb{P} \circ\left(\Delta S_{T}(\omega, \cdot)\right)^{-1}[A]=\mathbb{P}\left[\Delta S_{T}(\omega, \cdot) \in A\right],
$$

where $A \in \mathcal{B}\left(\mathbb{R}^{d}\right)$. We note that by definition,

$$
\mathbb{P} \circ\left(\Delta S_{T}(\omega, \cdot)\right)^{-1}\left[\tilde{\chi}_{\mathcal{F}_{T-1}^{0}}(\omega)\right]=1
$$

holds for all $\mathbb{P} \in \mathfrak{P}_{T-1}(\omega)$. With a slight abuse of notation, we recall the set

$$
B^{1}(\omega):=\left\{\omega^{\prime} \in \operatorname{proj}_{T}\left(\Sigma_{T-1}^{\omega} \cap \Omega^{\mathfrak{P}}\right): \xi_{T, \Omega^{\mathfrak{P}}}(\omega) \cdot \Delta S_{T}\left(\omega, \omega^{\prime}\right)>0\right\}
$$

from [13, proof of Lemma 1, Step 1] and note that for all $\mathbb{P} \in \mathfrak{P}_{T-1}(\omega)$,

$$
\begin{aligned}
& \mathbb{P}\left[\left\{\omega^{\prime} \in \operatorname{proj}_{T}\left(\Sigma_{T-1}^{\omega} \cap \Omega^{\mathfrak{P}}\right): \xi_{T, \Omega^{\mathfrak{P}}}(\omega) \cdot \Delta S_{T}\left(\omega, \omega^{\prime}\right)>0\right\}\right] \\
& =\mathbb{P} \circ\left(\Delta S_{T}(\omega, \cdot)\right)^{-1}\left[\left\{x \in \mathbb{R}^{d}: \xi_{T, \Omega^{\mathfrak{P}}}(\omega) \cdot x>0\right\}\right]
\end{aligned}
$$


follows. Clearly, the set $\left\{x \in \mathbb{R}^{d}: \xi_{T, \Omega \mathfrak{P}}(\omega) \cdot x>0\right\}$ is open in $\mathbb{R}^{d}$; thus by the definition of $\tilde{\chi}_{\mathcal{F}_{T-1}^{0}}(\omega)$, there is a $\tilde{\mathbb{P}} \in \mathfrak{P}_{T-1}(\omega)$ such that

$$
\tilde{\mathbb{P}}_{\Delta S_{T}(\omega, \cdot)}\left[\left\{x \in \mathbb{R}^{d}: \xi_{T, \Omega^{\mathfrak{P}}}(\omega) \cdot x>0\right\}\right]>0
$$

or there are no one-point arbitrages on $\Sigma_{T-1}^{\omega} \cap \Omega^{\mathfrak{P}}$. To finish the proof of 3) $\Rightarrow 1$ ), we need to select $\tilde{\mathbb{P}}$ in a measurable way, and this follows by standard arguments: Define the correspondence $\Psi: \mathbb{R}^{d} \times X_{T-1} \rightarrow \mathcal{P}\left(X_{1}\right)$ by

$$
\Psi(H, \omega)=\left\{\mathbb{P} \in \mathfrak{P}_{T-1}(\omega): \mathbb{E}_{\mathbb{P}}\left[H \cdot \Delta S_{T}(\omega, \cdot)\right]^{+}>0\right\} .
$$

This function has analytic graph by the arguments in [32, proof of Lemma 3.4]; so we can employ the Jankov-von-Neumann theorem (cf. [8, Proposition 7.49]) to find a universally measurable kernel

$$
\mathbb{P}_{T-1}^{\prime}: \mathbb{R}^{d} \times X_{T-1} \rightarrow \mathcal{P}\left(X_{1}\right)
$$

with the property that $\mathbb{P}_{T-1}^{\prime}(H, \omega) \in \mathfrak{P}_{T-1}(\omega)$ for all $(H, \omega) \in \mathbb{R}^{d} \times X_{T-1}$ and $\mathbb{P}_{T-1}^{\prime}(H, \omega) \in \Psi(H, \omega)$ on $\{\Psi(H, \omega) \neq \emptyset\}$. Then also the kernel

$$
\omega \mapsto \tilde{\mathbb{P}}_{T-1}(\omega):=\mathbb{P}_{T-1}^{\prime}\left(\xi_{T, \Omega \mathfrak{P}}(\omega), \omega\right)
$$

is universally measurable. Defining $\tilde{\mathbb{P}}:=\left.\hat{\mathbb{P}}\right|_{X_{T-1}} \otimes \tilde{\mathbb{P}}_{T-1}$, which is the product measure formed from the restriction of $\hat{\mathbb{P}}$ to $X_{T-1}$ and $\tilde{P}_{T-1}$, gives

$$
\tilde{\mathbb{P}}\left[\xi_{T, \Omega \mathfrak{P}} \cdot \Delta S_{T}>0\right]>0 .
$$

This proves 3 ) $\Rightarrow 1$ ) by backward induction.

Finally, we show 1$) \Rightarrow 2$ ): let us assume $\mathbb{P}\left[\left(\Omega^{\mathfrak{P}}\right)^{*}\right]=1$ for all $\mathbb{P} \in \mathfrak{P}$. Note that by the arguments given in the proof of 3 ) $\Rightarrow 1$ ), this means that

$$
\bigcup_{t=1}^{T}\left\{\omega \in \Omega^{\mathfrak{P}}: \text { there is a one-point arbitrage on } \Sigma_{t-1}^{\omega} \cap \Omega^{\mathfrak{P}}\right\}
$$

is a $\mathfrak{P}$-polar set, so in particular $0 \in \operatorname{ri}\left(\tilde{\chi}_{\mathcal{F}_{t-1}^{0}}(\omega)\right)$ for all $t=1, \ldots, T$ and $\mathfrak{P}$-q.e. $\omega \in X$. Here $\operatorname{ri}\left(\tilde{\chi}_{\mathcal{F}_{t-1}^{0}}(\omega)\right)$ denotes the relative interior of the convex hull of $\tilde{\chi}_{\mathcal{F}_{t-1}^{0}}(\omega)$. Let $\hat{\mathbb{P}} \in \mathfrak{P}$ be fixed. We define for an arbitrary $\mathbb{P} \in \mathfrak{P}$ and $\omega \in X_{t-1}$ the support of $\mathbb{P}_{t-1}(\omega) \circ\left(\Delta S_{t}(\omega, \cdot)\right)^{-1}$ conditioned on $\mathcal{F}_{t-1}^{0}$ as

$$
\chi_{\mathcal{F}_{t-1}^{0}}^{\mathbb{P}}(\omega)=\left\{x \in \mathbb{R}^{d}: \mathbb{P}_{t-1}(\omega)\left[\Delta S_{t}(\omega, \cdot) \in B_{\varepsilon}(x)\right]>0 \text { for all } \varepsilon>0\right\}
$$

Using selection arguments which are explained below, we can now find measurable selectors $\mathbb{P}_{(0,1)}, \ldots, \mathbb{P}_{(0, d)}, \mathbb{P}_{(1,1)}, \ldots, \mathbb{P}_{(T-1, d)}$ such that

$$
\mathbb{P}_{(t, 1)}(\omega), \ldots, \mathbb{P}_{(t, d)}(\omega) \in \mathfrak{P}_{t}(\omega)
$$


and $\mathbb{P}_{(0,1)}, \ldots, \mathbb{P}_{(T-1, d)}$ fulfil the following property: if we define

$$
\tilde{\mathbb{P}}_{t}(\omega)=\frac{1}{d+1}\left(\hat{\mathbb{P}}_{t}(\omega)+\sum_{i=1}^{d} \mathbb{P}_{(t, i)}(\omega)\right)
$$

for $t=0, \ldots, T-1$ and every $\omega \in X_{t}$, then for $\tilde{\mathbb{P}}=\tilde{\mathbb{P}}_{0} \otimes \cdots \otimes \tilde{\mathbb{P}}_{T-1}$, we have

$$
0 \in \operatorname{ri}\left(\chi_{\mathcal{F}_{t-1}^{0}}^{\tilde{\mathbb{P}}}\right) \quad \tilde{\mathbb{P}} \text {-a.s. for all } t=1, \ldots, T
$$

where $\operatorname{ri}\left(\chi_{\mathcal{F}_{t-1}^{0}}^{\tilde{\mathbb{P}}}\right)$ denotes the relative interior of the convex hull of $\chi_{\mathcal{F}_{t-1}^{0}}^{\tilde{\mathbb{P}}}$. We note that since $\mathfrak{P}_{t}(\omega)$ is convex, we have $\tilde{\mathbb{P}}_{t}(\omega) \in \mathfrak{P}_{t}(\omega)$ for $\omega \in X_{t}$, and by definition, $\hat{\mathbb{P}} \ll \tilde{\mathbb{P}}$ holds. Now it follows from [36, Theorem 1] that there exists a martingale measure $\mathbb{Q}$ equivalent to $\tilde{\mathbb{P}}$. The fact that $\tilde{\mathbb{P}} \in \mathfrak{P}$ implies $\mathbb{Q} \in \mathcal{Q}_{\mathfrak{P}}$, which shows the claim.

We now present the measurable selection argument: fix $t \in\{1, \ldots, T\}$. Note that for all $\omega \in \operatorname{proj}_{0: t-1}\left(\left(\Omega^{\mathfrak{P}}\right)^{*}\right)$, we get $0 \in \operatorname{ri}\left(\Delta S_{t}\left(\omega, \Sigma_{t-1}^{\omega} \cap\left(\Omega^{\mathfrak{P}}\right)^{*}\right)\right)$ by the definition of $\left(\Omega^{\mathfrak{P}}\right)^{*}$, which implies by $[11$, Theorem $\mathrm{D}]$ that there exist $\mathbb{P}^{1}, \ldots, \mathbb{P}^{d} \in \mathfrak{P}_{t-1}(\omega)$, which might not be pairwise distinct, such that

$$
0 \in \operatorname{ri}\left(\operatorname{supp}\left(\frac{\hat{\mathbb{P}}_{t-1}(\omega)+\mathbb{P}^{1}+\cdots+\mathbb{P}^{d}}{d+1} \circ\left(\Delta S_{t}(\omega, \cdot)\right)^{-1}\right)\right) .
$$

Note that $\omega \mapsto \hat{\mathbb{P}}_{t-1}(\omega)$ is universally measurable. We define the correspondence $\rho: \mathcal{P}\left(X_{1}\right)^{d+1} \rightarrow \mathbb{R}^{d}$ by

$$
\rho\left(\mathbb{P}^{0}, \mathbb{P}^{1}, \ldots, \mathbb{P}^{d}\right)=\operatorname{supp}\left(\frac{\mathbb{P}^{0}+\mathbb{P}^{1}+\cdots+\mathbb{P}^{d}}{d+1} \circ\left(\Delta S_{t}(\omega, \cdot)\right)^{-1}\right) .
$$

Note that for $O \subseteq \mathbb{R}^{d}$ open, we have

$$
\begin{aligned}
& \left\{\left(\mathbb{P}^{0}, \mathbb{P}^{1}, \ldots, \mathbb{P}^{d}\right): \rho\left(\mathbb{P}^{0}, \mathbb{P}^{1}, \ldots, \mathbb{P}^{d}\right) \cap O \neq \emptyset\right\} \\
& =\bigcup_{i=0}^{d}\left\{\left(\mathbb{P}^{0}, \mathbb{P}^{1}, \ldots, \mathbb{P}^{d}\right): \mathbb{P}^{i} \circ\left(\Delta S_{t}(\omega, \cdot)\right)^{-1}[O]>0\right\} .
\end{aligned}
$$

Since $\mathbb{P} \mapsto \mathbb{P}[O]$ is Borel-measurable, we conclude that $\rho$ is weakly measurable. Let us denote by $\mathbb{S}^{d}$ the unit sphere in $\mathbb{R}^{d}$. By preservation of measurability (cf. [35, Exercise 14.12]), it follows that the correspondence $\Psi: \mathcal{P}\left(X_{1}\right)^{d+1} \rightarrow \mathbb{R}^{d}$,

$$
\Psi\left(\mathbb{P}^{0}, \mathbb{P}^{1}, \ldots, \mathbb{P}^{d}\right)=\left\{H \in \mathbb{S}^{d}: H \cdot y \geq 0 \text { for all } y \in \rho\left(\mathbb{P}^{0}, \mathbb{P}^{1}, \ldots, \mathbb{P}^{d}\right)\right\}
$$

is weakly measurable. Then also the correspondence $\tilde{\Psi}: \mathcal{P}\left(X_{1}\right)^{d+1} \rightarrow \mathbb{R}^{d}$,

$$
\begin{aligned}
\tilde{\Psi}\left(\mathbb{P}^{0}, \mathbb{P}^{1}, \ldots, \mathbb{P}^{d}\right)= & \left\{H \in \mathbb{S}^{d}: H \cdot y \leq 0 \text { for all } y \in \rho\left(\mathbb{P}^{0}, \mathbb{P}^{1}, \ldots, \mathbb{P}^{d}\right)\right\} \\
& \cap \Psi\left(\mathbb{P}^{0}, \mathbb{P}^{1}, \ldots, \mathbb{P}^{d}\right)
\end{aligned}
$$


is weakly measurable and closed-valued. Let $V$ be a countable base of $\mathbb{R}^{d}$. The set

$$
\begin{aligned}
& \left\{\left(\mathbb{P}^{0}, \mathbb{P}^{1}, \ldots, \mathbb{P}^{d}\right): \tilde{\Psi}\left(\omega, \mathbb{P}^{1}, \ldots, \mathbb{P}^{d}\right)=\Psi\left(\mathbb{P}^{0}, \mathbb{P}^{1}, \ldots, \mathbb{P}^{d}\right)\right\} \\
& =\bigcap_{O: O \in V}\left(\left(\left\{\left(\mathbb{P}^{0}, \mathbb{P}^{1}, \ldots, \mathbb{P}^{d}\right): \Psi \cap O \neq \emptyset\right\} \cap\left\{\left(\mathbb{P}^{0}, \mathbb{P}^{1}, \ldots, \mathbb{P}^{d}\right): \tilde{\Psi} \cap O \neq \emptyset\right\}\right)\right. \\
& \left.\quad \cup\left(\left\{\left(\mathbb{P}^{0}, \mathbb{P}^{1}, \ldots, \mathbb{P}^{d}\right): \Psi \cap O=\emptyset\right\} \cap\left\{\left(\mathbb{P}^{0}, \mathbb{P}^{1}, \ldots, \mathbb{P}^{d}\right): \tilde{\Psi} \cap O=\emptyset\right\}\right)\right)
\end{aligned}
$$

is Borel-measurable. Note that for an arbitrary convex set $C \subseteq \mathbb{R}^{d}$, the relationship

$$
0 \in \operatorname{ri}(C) \Leftrightarrow\left(\forall H \in \mathbb{S}^{d} \text { such that } H \cdot x \geq 0, \forall x \in C \Rightarrow H \cdot x=0, \forall x \in C\right)
$$

holds. Let

$$
A:=\left\{\left(\mathbb{P}^{0}, \mathbb{P}^{1}, \ldots, \mathbb{P}^{d}\right): 0 \in \operatorname{ri}\left(\rho\left(\mathbb{P}^{0}, \mathbb{P}^{1}, \ldots, \mathbb{P}^{d}\right)\right) \text { for } i=1, \ldots, d\right\} .
$$

Then from the above arguments, it follows that $A$ is Borel, and in particular the setvalued mapping

$$
\begin{aligned}
& A\left(\omega, \mathbb{P}^{0}\right):=\left\{\left(\mathbb{P}^{1}, \ldots, \mathbb{P}^{d}\right):\right. 0 \in \operatorname{ri}\left(\rho\left(\mathbb{P}^{0}, \mathbb{P}^{1}, \ldots, \mathbb{P}^{d}\right)\right), \\
&\left.\mathbb{P}^{i} \in \mathfrak{P}_{t-1}(\omega) \text { for } i=1, \ldots, d\right\}
\end{aligned}
$$

has analytic graph. We can now employ the Jankov-von-Neumann theorem (cf. [8, Proposition 7.49]) to find universally measurable kernels $\mathbb{P}_{t-1}^{i}: X_{t-1} \rightarrow \mathcal{P}\left(X_{1}\right)$ such that for every $\omega \in X_{t-1}$, we have $\mathbb{P}_{t-1}^{i}(\omega) \in \mathfrak{P}_{t-1}(\omega)$ and

$$
0 \in \operatorname{ri}\left(\rho\left(\hat{\mathbb{P}}_{t-1}(\omega), \mathbb{P}_{t-1}^{1}, \ldots, \mathbb{P}_{t-1}^{d}\right)\right) .
$$

This concludes the proof of 1) $\Rightarrow 2$ ).

The second part of Theorem 2.5 follows immediately from Proposition 4.1.

Before continuing the proof of Theorem 2.5, let us first give a short remark on the measurability of the arbitrage strategies involved in the proof above.

Remark 4.5 By the FTAP of Burzoni et al. [13], there exists a filtration $\tilde{\mathbb{F}}$ satisfying $\mathbb{F}^{0} \subseteq \tilde{\mathbb{F}} \subseteq \mathbb{F}^{M}$ and such that there is no strong arbitrage in $\mathcal{H}(\tilde{\mathbb{F}})$ on $\Omega^{\mathfrak{P}}$. More concretely, there exists an arbitrage aggregator $H^{*}$ in $\mathcal{H}(\widetilde{\mathbb{F}})$ and thus in $\mathcal{H}\left(\mathbb{F}^{M}\right)$. So in particular if $\mathbb{P}\left[\Omega^{\mathfrak{P}} \backslash\left(\Omega^{\mathfrak{P}}\right)^{*}\right]>0$ for some $\mathbb{P} \in \mathfrak{P}$, then $H^{*}$ is a $\mathfrak{P}$-q.s. arbitrage in $\mathcal{H}(\tilde{\mathbb{F}})$. In general, the inclusion $\tilde{\mathbb{F}} \subseteq \mathbb{F}^{\mathcal{U}}$ does not hold. This is why we need to construct a new $\mathbb{F}^{\mathcal{U}}$-measurable arbitrage strategy which captures the arbitrages essential for $\mathfrak{P}$. More generally, in this paper, we manage to avoid using projectively measurable sets which were essential for the arguments in [13]. In fact, all our trading strategies are universally measurable without invoking the axiom of projective determinacy.

Furthermore, we hope that by constructing an explicit arbitrage strategy in the proof of 3$) \Rightarrow 1$ ) above clarifies and improves the reasoning in Burzoni et al. [14, Theorem 4.23] (in particular [14, A.3]), as our arguments apply to the particular case 
$\mathfrak{P}=\{\mathbb{P}\}$ discussed there (and our reasoning can even be significantly simplified in this case). Introducing a measurable separator $\xi$, it is apparent that $j_{z}$ in [14, A.3] can always be chosen equal to one in our setting. Also the resulting strategy $H^{\mathbb{P}}$ therein can be chosen universally measurable.

To prove the first part of Theorem 2.5 for the case $\Phi \neq 0$, we recall the following notion from Burzoni et al. [13].

Definition 4.6 ([13, Definition 4]) A pathspace partition scheme $\mathcal{R}\left(\alpha^{*}, H^{*}\right)$ of $\Omega$ is a collection of trading strategies $H_{1}, \ldots, H_{\beta} \in \mathcal{H}\left(\mathbb{F}^{\mathcal{U}}\right), \alpha_{1}, \ldots, \alpha_{\beta} \in \mathbb{R}^{k}$ and arbitrage aggregators $\tilde{H}_{0}, \ldots, \tilde{H}_{\beta}$ for some $1 \leq \beta \leq k$ such that

1) the vectors $\alpha_{i}, i=1, \ldots, \beta$, are linearly independent;

2) for any $i \leq \beta$,

$$
\alpha_{i} \cdot \Phi+H_{i} \cdot S_{T} \geq 0 \quad \text { on } A_{i-1}^{*},
$$

where $A_{0}=\Omega, A_{i}:=\left\{\alpha_{i} \cdot \Phi+H_{i} \cdot S_{T}=0\right\} \cap A_{i-1}^{*}$;

3) for any $i=0, \ldots, \beta, \tilde{H}_{i}$ is an arbitrage aggregator for $A_{i}$;

4) if $\beta<k$, then either $A_{\beta}=\emptyset$ or for any $\alpha \in \mathbb{R}^{k}$ linearly independent from $\alpha_{1}, \ldots, \alpha_{\beta}$, there does not exist $H$ such that

$$
\alpha \cdot \Phi+\left(H \cdot S_{T}\right) \geq 0 \quad \text { on } A_{\beta}^{*} .
$$

Definition $4.7\left(\left[13\right.\right.$, Definition 5]) A pathspace partition scheme $\mathcal{R}\left(\alpha^{*}, H^{*}\right)$ is successful if $A_{\beta}^{*} \neq \emptyset$.

We quote the following results.

Lemma $4.8\left(\left[13\right.\right.$, Lemma 5]) For any $\mathcal{R}\left(\alpha^{*}, H^{*}\right)$, we have $A_{i}^{*}=\Omega_{\left\{\alpha^{j} . \Phi: j \leq i\right\}}^{*}$. Moreover, if $\mathcal{R}\left(\alpha^{*}, H^{*}\right)$ is successful, then $A_{\beta}^{*}=\Omega_{\Phi}^{*}$.

Lemma 4.9 ([13, proof of Theorem 1 for $\Phi \neq 0]$ ) A pathspace partition scheme $\mathcal{R}\left(\alpha^{*}, H^{*}\right)$ is successful if and only if $\Omega_{\Phi}^{*} \neq \emptyset$.

We now complete the first part of the proof of Theorem 2.5 for the case $\Phi \neq 0$.

Proof of Theorem 2.5 for $\Phi \neq 0$ The existence of $\Omega^{\mathfrak{P}}$ and 1) $\Rightarrow$ no strong arbitrage in $\mathcal{A}_{\Phi}(\tilde{\mathbb{F}})$ on $\Omega^{\mathfrak{P}}$ as well as 2$) \Rightarrow 3$ ) follow exactly as before. Following closely the ideas of $[12$, Theorem 5.1], we now argue that 1$) \Rightarrow 2$ ) holds by induction over the number $e$ of options available for static trading. In particular, we can assume without loss of generality that there exists a random variable $\varphi \geq 1$ such that $\left|\phi_{j}\right| \leq \varphi$ for all $j=1, \ldots, k$ and consider the set $\mathcal{Q}_{\varphi}=\left\{\mathbb{Q} \in \mathcal{Q}_{\mathfrak{P}}: \mathbb{E}_{\mathbb{Q}}[\varphi]<\infty\right\}$ in order to avoid integrability issues. So let us assume there are $e \geq 0$ traded options $\phi_{1}, \ldots, \phi_{e}$, for which 1) $\Rightarrow 2$ ) holds. We introduce an additional option $g=\phi_{e+1}$ and assume $\mathbb{P}\left[\left(\Omega^{\mathfrak{P}}\right)_{\left\{\phi_{1}, \ldots, \phi_{e+1}\right\}}^{*}\right]=1$ for all $\mathbb{P} \in \mathfrak{P}$. Then clearly $\mathbb{P}\left[\left(\Omega^{\mathfrak{P}}\right)_{\left\{\phi_{1}, \ldots, \phi_{e}\right\}}^{*}\right]=1$ for all $\mathbb{P} \in \mathfrak{P}$, and by the induction hypothesis, there is no arbitrage in the market with 
options $\left\{\phi_{1}, \ldots, \phi_{e}\right\}$ available for static trading. Let $\mathbb{P} \in \mathfrak{P}$. Then by exactly the same arguments as in [12, proof of Theorem 5.1(a)], we can use convexity of $\mathcal{Q}_{\varphi}$ and Theorem 2.9 to find a measure $\mathbb{Q} \in \mathcal{Q}_{\varphi}$ such that $\mathbb{P} \ll \mathbb{Q}$ and $\mathbb{Q} \in \mathcal{Q}_{\mathfrak{P},\left\{\phi_{1}, \ldots, \phi_{e+1}\right\}}$; so 2) holds.

Finally, it remains to show 3$) \Rightarrow 1$ ). Let us thus assume there exists $\hat{\mathbb{P}} \in \mathfrak{P}$ such that $\hat{\mathbb{P}}\left[\Omega^{\mathfrak{P}} \backslash\left(\Omega^{\mathfrak{P}}\right)_{\Phi}^{*}\right]>0$. We want to find $(h, H) \in \mathcal{A}_{\Phi}(\mathbb{F} \mathcal{U})$ and $\tilde{\mathbb{P}} \in \mathfrak{P}$ such that $h \cdot \Phi+H \cdot S_{T} \geq 0 \mathfrak{P}$-q.s. and $\tilde{\mathbb{P}}\left[h \cdot \Phi+H \cdot S_{T}>0\right]>0$. We use the properties of a pathspace partition scheme $\mathcal{R}\left(\alpha^{*}, H^{*}\right)$ recalled above. We define

$$
\begin{aligned}
& m=\min \left\{k \in\{0, \ldots, \beta\}: \tilde{\mathbb{P}}\left[A_{k} \backslash A_{k}^{*}\right]>0 \text { for some } \tilde{\mathbb{P}} \in \mathfrak{P}\right\}, \\
& \tilde{m}=\min \left\{k \in\{1, \ldots, \beta\}: \tilde{\mathbb{P}}\left[A_{k-1}^{*} \backslash A_{k}\right]>0 \text { for some } \tilde{\mathbb{P}} \in \mathfrak{P}\right\},
\end{aligned}
$$

where $A_{0}=\Omega^{\mathfrak{P}}$. If $\tilde{m} \leq m$, we select the strategy $\left(\alpha_{\tilde{m}}, H_{\tilde{m}}\right) \in \mathcal{A}_{\Phi}\left(\mathbb{F}^{\mathcal{U}}\right)$ which satisfies $H_{\tilde{m}} \cdot S_{T}+\alpha_{\tilde{m}} \cdot \Phi \geq 0$ on $A_{\tilde{m}-1}^{*}$. We note that $\mathbb{P}\left[A_{\tilde{m}-1}^{*}\right]=1$ for all $\mathbb{P} \in \mathfrak{P}$ by the definition of $m, \tilde{m}$ and that $\left\{H_{\tilde{m}} \bullet S_{T}+\alpha_{\tilde{m}} \cdot \Phi>0\right\}=A_{\tilde{m}-1}^{*} \backslash A_{\tilde{m}}$ so that $\tilde{\mathbb{P}}\left[H_{\tilde{m}} \bullet S_{T}+\alpha_{\tilde{m}} \cdot \Phi>0\right]>0$ for some $\tilde{\mathbb{P}} \in \mathfrak{P}$. If $\tilde{m}>m$, then $\mathbb{P}\left[A_{m}\right]=1$ for all $\mathbb{P} \in \mathfrak{P}$ and $\tilde{\mathbb{P}}\left[A_{m} \backslash A_{m}^{*}\right]>0$ for some $\tilde{\mathbb{P}} \in \mathfrak{P}$; so we can argue as in the proof of Proposition 2.5 for $\Phi=0,3) \Rightarrow 1$ ), using a standard separator and measurable selection of a measure in $\mathfrak{P}$.

As before, the second part of Theorem 2.5 follows immediately from Proposition 4.1. This concludes the proof.

Proof of Theorem 2.7 We recall the analytic set $\Omega^{\mathfrak{P}}$ from the proof of Theorem 2.5 for $\Phi=0$ and the sets $\left(C_{n}\right)_{n \in \mathbb{N}}$ from (2.1). Now we define $B \in \mathcal{B}(X)$ by

$$
B:=\text { union over all } C_{n}, n \in \mathbb{N} \text {, with } \mathbb{P}\left[C_{n} \cap\left(\Omega^{\mathfrak{P}}\right)_{\Phi}^{*}\right]=0 \text { for all } \mathbb{P} \in \mathfrak{P} \text {. }
$$

We claim that (2.1) implies that

$$
\begin{aligned}
& B \cap\left(\Omega^{\mathfrak{P}}\right)_{\Phi}^{*} \\
& =\left(\text { union over all } C \in \mathcal{S} \text { with } \mathbb{P}\left[C \cap\left(\Omega^{\mathfrak{P}}\right)_{\Phi}^{*}\right]=0 \text { for all } \mathbb{P} \in \mathfrak{P}\right) \cap\left(\Omega^{\mathfrak{P}}\right)_{\Phi}^{*} \\
& =\left(\text { union over all } C \in \mathcal{S} \text { with } C \cap\left(\Omega^{\mathfrak{P}}\right)_{\Phi}^{*} \in \mathcal{N}^{\mathfrak{P}}\right) \cap\left(\Omega^{\mathfrak{P}}\right)_{\Phi}^{*} .
\end{aligned}
$$

Indeed, clearly $B$ is contained in $\widetilde{B}:=$ union over all $C \in \mathcal{S}$ with $C \cap\left(\Omega^{\mathfrak{P}}\right)_{\Phi}^{*} \in \mathcal{N}^{\mathfrak{P}}$. Now assume towards a contradiction that there exists

$$
\omega \in\left(\widetilde{B} \cap\left(\Omega^{\mathfrak{P}}\right)_{\Phi}^{*}\right) \backslash\left(B \cap\left(\Omega^{\mathfrak{P}}\right)_{\Phi}^{*}\right)
$$

In particular, $\omega \in C$ for some $C \in \mathcal{S}$ such that $C \cap\left(\Omega^{\mathfrak{P}}\right)_{\Phi}^{*} \in \mathcal{N}^{\mathfrak{P}}$. By (2.1), there exists $n_{0} \in \mathbb{N}$ such that $C_{n_{0}} \subseteq C$ and $\omega \in C_{n_{0}}$. This implies $\omega \in B$ and thus shows the claim. Let us now first assume that $\Phi=0$ and set

$$
\Omega:=\Omega^{\mathfrak{P}} \backslash\left(\left(\Omega^{\mathfrak{P}}\right)^{*} \cap B\right) \in \mathcal{F}^{\mathcal{U}} .
$$


By assumption, we have $\mathbb{P}[\Omega]=1$ for all $\mathbb{P} \in \mathfrak{P}$. By the definition of the operation $(\cdot)^{*}$,

$$
\Omega^{*}=\left(\left(\Omega^{\mathfrak{P}}\right)^{*} \backslash B\right)_{\Phi}^{*}=\left(\Omega^{\mathfrak{P}} \backslash B\right)^{*}
$$

follows. To see the above equality, take a martingale measure $\mathbb{Q} \in \mathcal{M}_{\Omega, \Phi}$ and assume that $\mathbb{Q}\left[\Omega \backslash\left(\Omega^{\mathfrak{P}} \backslash B\right)\right]>0$. As $\Omega \backslash\left(\Omega^{\mathfrak{P}} \backslash B\right)=\Omega^{\mathfrak{P}} \backslash\left(\Omega^{\mathfrak{P}}\right)^{*} \cap B$, we conclude that $\mathbb{Q}\left[\Omega^{\mathfrak{P}} \backslash\left(\Omega^{\mathfrak{P}}\right)^{*}\right]>0$. Since any calibrated martingale measure supported on a subset of $\Omega$ is in $\mathcal{M}_{\Omega^{\mathfrak{P}}}$, this leads to a contradiction to the definition of $\left(\Omega^{\mathfrak{P}}\right)^{*}$. Also, $\Omega^{\mathfrak{P}} \backslash B=\Omega^{\mathfrak{P}} \cap B^{c}$ is the intersection of two analytic sets; so we conclude that $\Omega^{*}$ is analytic. Finally, by the definition of $\Omega^{\mathfrak{P}}$, we conclude that $\Omega^{*}=\left(\Omega^{\mathfrak{P}}\right)^{*} \mathfrak{P}$-q.s.

The implications 1$) \Rightarrow 2$ ) $\Rightarrow 3$ ) $\Rightarrow 4$ ) $\Rightarrow$ 5) follow directly from the definition. Thus we only need to show (5) $\Rightarrow 1$ ). Let us fix $C \in \mathcal{S}$ such that $C \subseteq \Omega$. No arbitrage de la classe $\mathcal{S}$ on $\Omega$ implies that $\Omega^{*} \cap C \neq \emptyset$. From (4.1), we thus conclude that $\mathbb{P}\left[\left(\Omega^{\mathfrak{P}}\right)^{*} \cap C\right]>0$ for some $\mathbb{P} \in \mathfrak{P}$. As $\Omega^{*}=\left(\Omega^{\mathfrak{P}}\right)^{*} \mathfrak{P}$-q.s., this implies $\mathbb{P}\left[\Omega^{*} \cap C\right]>0$. Using a construction similar to the proof of Proposition 2.5 for the case $\Phi=0$, we can find a measure $\tilde{\mathbb{P}} \in \mathfrak{P}$ such that $\tilde{\mathbb{P}}[C]>0$ and 0 is in the interior of the conditional support of $\tilde{\mathbb{P}}\left[\cdot \mid \Omega^{*}\right]$. By $[36$, Theorem 1$]$, we conclude that there exists a martingale measure $\mathbb{Q} \in \mathcal{Q}_{\mathfrak{P}}$ equivalent to $\tilde{\mathbb{P}}\left[\cdot \mid \Omega^{*}\right]$; in particular, $\mathbb{Q}[C]>0$. The case $\Phi \neq 0$ can now be treated similarly; indeed, we define $\Omega^{\mathfrak{P}, \Phi}$ as in the proof of Theorem 2.5 for $\Phi=0$, but now including the statically traded options $\Phi$ in the definition of the quasi-sure support, and we follow the same arguments as above. This concludes the proof.

\subsection{Proof of Theorem 2.9}

We first show that the quasi-sure superhedging theorem of Bouchard and Nutz [12] implies the second part of Theorem 2.9.

Proposition 4.10 Let $\Omega$ be an analytic subset of $X$ and $\Omega_{\Phi}^{*} \neq \emptyset$. Let the set $\mathfrak{P}$ satisfy (APS) and $\mathcal{N}^{\mathfrak{P}}=\mathcal{N}^{\mathcal{M}_{\Omega, \Phi}^{f}}$ Then $\mathcal{N}^{\mathcal{Q}_{\mathfrak{P}, \Phi}}=\mathcal{N}^{\mathcal{M}_{\Omega, \Phi}^{f}}$, and for any upper semianalytic function $g: X \rightarrow \mathbb{R}$,

$$
\sup _{\mathbb{Q} \in \mathcal{M}_{\Omega, \Phi}^{f}} \mathbb{E}_{\mathbb{Q}}[g]=\pi_{\Omega_{\Phi}^{*}}(g)=\pi^{\mathfrak{P}}(g)=\sup _{\mathbb{Q} \in \mathcal{Q}_{\mathfrak{P}, \Phi}} \mathbb{E}_{\mathbb{Q}}[g] .
$$

Proof That $\mathcal{Q}_{\mathfrak{P}, \Phi}$ and $\mathcal{M}_{\Omega, \Phi}^{f}$ have the same polar sets follows by the definition of $\Omega_{\Phi}^{*}$ and [13, Lemma 2]. We now show (4.2): consider

$$
\mathfrak{P}^{\Omega}:=\mathcal{P}^{f}\left(\Omega_{\Phi}^{*}\right)
$$

Note that there is no $\mathcal{M}_{\Omega \text {, } \Phi}^{f}$-q.s. arbitrage if and only if there is no $\mathfrak{P}^{\Omega}$-q.s. arbitrage.

We now show that $\Omega_{\Phi}^{*}$ is analytic if $\Omega$ is analytic. Recall the set $\mathcal{P}_{Z, \Phi}$ from Burzoni et al. [15, Lemma 5.4], defined by

$$
\mathcal{P}_{Z, \Phi}:=\left\{\mathbb{P} \in \mathcal{P}^{f}(X): \exists \mathbb{Q} \in \mathcal{M}_{X, \Phi}^{f} \text { such that } \frac{d \mathbb{Q}}{d \mathbb{P}}=\frac{c(\mathbb{P})}{1+Z}\right\},
$$


where $Z=\max _{i=1, \ldots, d} \max _{t=0, \ldots, T}\left|S_{t}^{i}\right|$ and $c(\mathbb{P})=\left(\mathbb{E}_{\mathbb{P}}[1+Z]^{-1}\right)^{-1}$. Now Burzoni et al. [15] show that the set $\left\{(\omega, \mathbb{P}): \omega \in X^{*}, \mathbb{P} \in \mathcal{P}^{\omega}\right\}$ is analytic, where $\mathcal{P}^{\omega}=\left\{\mathbb{P} \in \mathcal{P}_{Z, \Phi}: \mathbb{P}[\{\omega\}]>0\right\}$. Note that $\left\{(\omega, \mathbb{P}): \omega \in X^{*}, \mathbb{P} \in \mathcal{P}^{\omega}\right\} \cap\left(\Omega \times \mathcal{P}^{f}(\Omega)\right)$ is analytic and the projection of the above set to the first coordinate is exactly $\Omega_{\Phi}^{*}$, which shows that $\Omega_{\Phi}^{*}$ is analytic. We note that $\omega \mapsto \mathfrak{P}_{t}^{\Omega}(\omega)=\mathcal{P}^{f}\left(\operatorname{proj}_{t+1}\left(\Sigma_{t}^{\omega} \cap \Omega_{\Phi}^{*}\right)\right)$ has an analytic graph by exactly the same argument as in the proof of Proposition 4.1, replacing $\Omega$ by $\Omega_{\Phi}^{*}$. The result now follows from the superhedging theorem of [12] and the definition of $\mathcal{M}_{\Omega, \Phi}^{f}$.

We now show that the classical $\mathbb{P}$-a.s. one-step superhedging duality can be deduced by means of pathwise reasoning.

Lemma 4.11 Let $t \in\{0, \ldots, T-1\}$ and $g: X_{t+1} \rightarrow \mathbb{R}$ be $\mathcal{F}_{t+1}^{\mathcal{U}}$-measurable. Let $\mathbb{P} \in \mathcal{P}\left(X_{1}\right)$ and fix $\omega \in X_{t}$ such that $N A(\mathbb{P})$ holds for the one-period model $\left(S_{t}(\omega), S_{t+1}(\omega, \cdot)\right)$. Then

$$
\begin{aligned}
& \sup \left\{\mathbb{E}_{\mathbb{Q}}[g(\omega, \cdot)]: \mathbb{Q} \approx \mathbb{P}, \mathbb{Q} \in \mathcal{M}_{X_{1}}\right\} \\
& =\inf \left\{x \in \mathbb{R}: \exists H \in \mathbb{R}^{d} \text { such that } x+H \Delta S_{t+1}(\omega, \cdot) \geq g(\omega, \cdot) \mathbb{P} \text {-a.s. }\right\} .
\end{aligned}
$$

Proof As $g$ is $\mathcal{F}_{t+1}^{\mathcal{U}}$-measurable, there exists by [8, Lemma 7.27] a Borel-measurable function $\tilde{g}:\left(\mathbb{R}^{d}\right)^{t+1} \rightarrow \mathbb{R}$ such that $g(\omega)=\tilde{g}\left(S_{0: t+1}(\omega)\right)$ for $\mathbb{P}$-a.e. $\omega \in X_{t+1}$. Assume first that $S_{t+1} \mapsto \tilde{g}\left(S_{0: t}(\omega), S_{t+1}\right)$ is continuous and define the set

$$
\chi^{\mathbb{P}}:=\operatorname{supp}\left(\mathbb{P} \circ\left(\Delta S_{t+1}(\omega, \cdot)\right)^{-1}\right) .
$$

Then $\chi^{\mathbb{P}}=\left(\chi^{\mathbb{P}}\right)^{*}$ as NA(P) holds, and thus by [13, Theorem 2] and continuity of $S_{t+1} \mapsto \tilde{g}\left(S_{0: t}(\omega), S_{t+1}\right)$ as well as $S_{t+1} \mapsto H\left(S_{t+1}-S_{t}(\omega)\right)$,

$$
\begin{aligned}
& \sup \left\{\mathbb{E}_{\mathbb{Q}}[g(\omega, \cdot)]: \mathbb{Q} \approx \mathbb{P}, \mathbb{Q} \in \mathcal{M}_{X_{1}}\right\} \\
& \leq \inf \left\{x \in \mathbb{R}: \exists H \in \mathbb{R}^{d} \text { such that } x+H \Delta S_{t+1}(\omega, \cdot) \geq g(\omega, \cdot) \mathbb{P} \text {-a.s. }\right\} \\
& =\inf \left\{x \in \mathbb{R}: \exists H \in \mathbb{R}^{d} \text { such that } x+H \Delta S_{t+1}(\omega, \cdot) \geq \tilde{g}\left(S_{0: t}(\omega), \cdot\right) \text { on } \chi^{\mathbb{P}}\right\} \\
& =\sup \left\{\mathbb{E}_{\mathbb{Q}}\left[\tilde{g}\left(S_{0: t}(\omega), \cdot\right)\right]: \mathbb{Q} \in \mathcal{M}_{\chi^{\mathbb{P}}}^{f}\right\} \\
& \leq \sup \left\{\mathbb{E}_{\mathbb{Q}}\left[\tilde{g}\left(S_{0: t}(\omega), \cdot\right)\right]: \mathbb{Q} \approx \mathbb{P}_{0}\left(\Delta S_{t+1}(\omega, \cdot)\right)^{-1}, \mathbb{Q} \in \mathcal{M}_{\mathbb{R}^{d}}\right\} \\
& =\sup \left\{\mathbb{E}_{\mathbb{Q}}[g(\omega, \cdot)]: \mathbb{Q} \approx \mathbb{P}, \mathbb{Q} \in \mathcal{M}_{X_{1}}\right\} .
\end{aligned}
$$

If $S_{t+1} \mapsto \tilde{g}\left(S_{0: t}(\omega), S_{t+1}\right)$ is Borel-measurable, then by Lusin's theorem, there exists an increasing sequence $\left(K_{n}\right)_{n \in \mathbb{N}}$ of compact sets such that

$$
K_{n} \subseteq \chi^{\mathbb{P}}, \quad \mathbb{P} \circ\left(\Delta S_{t+1}(\omega, \cdot)\right)^{-1}\left[K_{n}^{c}\right] \leq 1 / n
$$


and $\left.\tilde{g}\left(S_{0: t}(\omega), \cdot\right)\right|_{K_{n}}$ is continuous. In particular, there exists $n_{0} \in \mathbb{N}$ such that for all $n \geq n_{0}$, we have $K_{n}=\left(K_{n}\right)^{*}$. By the above argument,

$$
\begin{aligned}
& \inf \left\{x \in \mathbb{R}: \exists H \in \mathbb{R}^{d} \text { such that } x+H \Delta S_{t+1}(\omega, \cdot) \geq \tilde{g}\left(S_{0: t}(\omega), \cdot\right) \text { on } K_{n}\right\} \\
& =\sup \left\{\mathbb{E}_{\mathbb{Q}}[g(\omega, \cdot)]: \mathbb{Q} \approx \mathbb{P} \circ\left(\Delta S_{t+1}(\omega, \cdot)\right)^{-1}, \mathbb{Q} \in \mathcal{M}_{K_{n}}\right\}
\end{aligned}
$$

holds for $n \geq n_{0}$. The claim now follows by taking suprema in $n \in \mathbb{N}$ on both sides of (4.3); indeed,

$$
\begin{aligned}
& \inf \left\{x \in \mathbb{R}: \exists H \in \mathbb{R}^{d} \text { such that } x+H \Delta S_{t+1}(\omega, \cdot) \geq g(\omega, \cdot) \mathbb{P} \text {-a.s. }\right\} \\
& =\sup _{n \in \mathbb{N}} \inf \left\{x \in \mathbb{R}: \exists H \in \mathbb{R}^{d} \text { such that } x+H \Delta S_{t+1}(\omega, \cdot) \geq \tilde{g}\left(S_{0: t}(\omega), \cdot\right) \text { on } K_{n}\right\} \\
& =\sup _{n \in \mathbb{N}} \sup \left\{\mathbb{E}_{\mathbb{Q}}\left[\tilde{g}\left(S_{0: t}(\omega), \cdot\right)\right]: \mathbb{Q} \approx \mathbb{P} \circ\left(\Delta S_{t+1}(\omega, \cdot)\right)^{-1}, \mathbb{Q} \in \mathcal{M}_{K_{n}}\right\} \\
& \leq \sup \left\{\mathbb{E}_{\mathbb{Q}}\left[\tilde{g}\left(S_{0: t}(\omega), \cdot\right)\right]: \mathbb{Q} \approx \mathbb{P} \circ\left(\Delta S_{t+1}(\omega, \cdot)\right)^{-1}, \mathbb{Q} \in \mathcal{M}_{\mathbb{R}^{d}}\right\} \\
& \leq \inf \left\{x \in \mathbb{R}: \exists H \in \mathbb{R}^{d} \text { such that } x+H \Delta S_{t+1}(\omega, \cdot) \geq g(\omega, \cdot) \mathbb{P} \text {-a.s. }\right\} .
\end{aligned}
$$

Using this one-step duality result under fixed $\mathbb{P}$ and (APS) of $\mathfrak{P}$, we now prove the first part of Theorem 2.9, which is restated in the following proposition.

Proposition 4.12 Let NA(P) hold and let $g: X \rightarrow \mathbb{R}$ be upper semianalytic. Then there exist a measure $\mathbb{P}^{g}=\mathbb{P}_{0}^{g} \otimes \cdots \otimes \mathbb{P}_{T-1}^{g}$ and an $\mathcal{F}^{\mathcal{U}}$-measurable set $\Omega_{g}^{\mathfrak{P}}$ with $\mathbb{P}\left[\Omega_{g}^{\mathfrak{P}}\right]=1$ for all $\mathbb{P} \in \mathfrak{P}$ such that

$$
\pi^{\mathfrak{P}}(g)=\pi^{\hat{\mathbb{P}}}(g)=\pi_{\left(\Omega_{g}^{\mathfrak{P}}\right)_{\Phi}^{*}}(g)=\sup _{\mathbb{Q} \in \mathcal{M}_{\Omega_{g}^{\mathfrak{P}}, \Phi}} \mathbb{E}_{\mathbb{Q}}[g]=\sup _{\mathbb{Q} \in \mathcal{Q}_{\mathfrak{P}, \Phi}} \mathbb{E}_{\mathbb{Q}}[g] .
$$

Proof We note that by $\mathbf{N A}(\mathfrak{P})$ and Theorem 2.5 , the set difference $\Omega^{\mathfrak{P}} \backslash\left(\Omega^{\mathfrak{P}}\right)_{\Phi}^{*}$ is $\mathfrak{P}$-polar. We first take $\Phi=0$. Recall the definition of the one-step functionals given in [12, Lemma 4.10] as

$$
\begin{aligned}
\mathcal{E}_{T}(g)(\omega) & =g(\omega), \\
\mathcal{E}_{t}(g)(\omega) & =\sup _{\mathbb{Q} \in \mathcal{Q}_{t}(\omega)} \mathbb{E}_{\mathbb{Q}}\left[\mathcal{E}_{t+1}(g)(\omega, \cdot)\right], \quad t=0, \ldots, T-1 .
\end{aligned}
$$

By (APS) and upper semianalyticity of $g$, every $\mathcal{E}_{t}(g)$ is upper semianalytic. We show recursively that for every $t=0, \ldots, T-1$ and for $\mathfrak{P}$-q.e. $\omega \in X_{t}$, there exists a measure $\mathbb{P} \in \mathcal{P}\left(X_{1}\right)$ such that $\mathbf{N A}(\mathbb{P})$ holds and

$$
\sup _{\mathbb{Q} \in \mathcal{Q}_{t}(\omega)} \mathbb{E}_{\mathbb{Q}}\left[\mathcal{E}_{t+1}(g)(\omega, \cdot)\right]=\sup _{\mathbb{Q} \approx \mathbb{P}, \mathbb{Q} \in \mathcal{M}_{X_{1}}} \mathbb{E}_{\mathbb{Q}}\left[\mathcal{E}_{t+1}(g)(\omega, \cdot)\right] .
$$

Note that by measurable selection arguments and the construction of $\Omega^{\mathfrak{P}}$, we conclude that for $\mathfrak{P}$-q.e. $\omega \in X_{t}$, the properties $\mathbf{N A}\left(\mathfrak{P}_{t}(\omega)\right)$ and $\mathbb{P}\left[\operatorname{proj}_{t+1}\left(\Omega^{\mathfrak{P}} \cap \Sigma_{t}^{\omega}\right)\right]=1$ 
hold for all $\mathbb{P} \in \mathfrak{P}_{t}(\omega)$. We now fix $t \in\{0, \ldots, T-1\}$ and $\omega \in X_{t}$ such that $\mathbf{N A}\left(\mathfrak{P}_{t}(\omega)\right)$ and $\mathbb{P}\left[\operatorname{proj}_{t+1}\left(\Omega^{\mathfrak{P}} \cap \Sigma_{t}^{\omega}\right)\right]=1$ for all $\mathbb{P} \in \mathfrak{P}_{t}(\omega)$ hold. Note that there exists a sequence $\left(\mathbb{P}_{n}\right)_{n \in \mathbb{N}}$ such that $\mathbb{P}_{n} \in \mathfrak{P}_{t}(\omega)$ for all $n \in \mathbb{N}$ and

$$
\sup _{\mathbb{Q} \ll \mathbb{P}_{n}, \mathbb{Q} \in \mathcal{M}_{X_{1}}} \mathbb{E}_{\mathbb{Q}}\left[\mathcal{E}_{t+1}(g)(\omega, \cdot)\right] \uparrow \sup _{\mathbb{Q} \in \mathcal{Q}_{t}(\omega)} \mathbb{E}_{\mathbb{Q}}\left[\mathcal{E}_{t+1}(g)(\omega, \cdot)\right] \quad \text { as } n \rightarrow \infty .
$$

We see from the proof of Theorem 2.5 for $\Phi=0$ in Sect. 4.1 that under $\mathbf{N A}\left(\mathfrak{P}_{t}(\omega)\right)$ and for a fixed $\mathbb{P} \in \mathfrak{P}_{t}(\omega)$, we can always find $\tilde{\mathbb{P}} \in \mathfrak{P}_{t}(\omega)$ such that $\mathbb{P} \ll \tilde{\mathbb{P}}$ and $\mathbf{N A}(\tilde{\mathbb{P}})$ holds. Thus we can assume without loss of generality that $\mathbf{N A}\left(\mathbb{P}_{n}\right)$ holds for all $n \in \mathbb{N}$. Define $\hat{\mathbb{P}}_{n}:=\sum_{k=1}^{n} 2^{-k} /\left(1-2^{-n}\right) \mathbb{P}_{k} \in \mathfrak{P}_{t}(\omega)$ as well as $\mathbb{P}_{t}^{g}(\omega):=\sum_{k=1}^{\infty} 2^{-k} \mathbb{P}_{k}$ and note that $\mathbf{N A}\left(\hat{\mathbb{P}}_{n}\right)$ as well as $\mathbf{N A}\left(\mathbb{P}_{t}^{g}(\omega)\right)$ hold for all $n \in \mathbb{N}$. Furthermore,

$$
\begin{aligned}
& \mathcal{E}_{t}(g)(\omega) \\
& =\sup _{n \in \mathbb{N}} \sup \left\{\mathbb{E}_{\mathbb{Q}}\left[\mathcal{E}_{t+1}(g)(\omega, \cdot)\right]: \mathbb{Q} \ll \mathbb{P}_{n}, \mathbb{Q} \in \mathcal{M}_{X_{1}}\right\} \\
& \leq \sup _{n \in \mathbb{N}} \sup \left\{\mathbb{E}_{\mathbb{Q}}\left[\mathcal{E}_{t+1}(g)(\omega, \cdot)\right]: \mathbb{Q} \approx \hat{\mathbb{P}}_{n}, \mathbb{Q} \in \mathcal{M}_{X_{1}}\right\} \\
& \leq \sup \left\{\mathbb{E}_{\mathbb{Q}}\left[\mathcal{E}_{t+1}(g)(\omega, \cdot)\right]: \mathbb{Q} \approx \mathbb{P}_{t}^{g}(\omega), \mathbb{Q} \in \mathcal{M}_{X_{1}}\right\} \\
& =\inf \left\{x \in \mathbb{R}: \exists H \in \mathbb{R}^{d} \text { with } x+H \Delta S_{t+1}(\omega, \cdot) \geq \mathcal{E}_{t+1}(g)(\omega, \cdot) \mathbb{P}_{t}^{g}(\omega) \text {-a.s. }\right\},
\end{aligned}
$$

where the last equality follows from Lemma 4.11. Define

$$
\begin{aligned}
& \pi_{t}^{\mathbb{P}_{t}^{g}(\omega)}(g)=\inf \left\{x \in \mathbb{R}: \exists H \in \mathbb{R}^{d}\right. \text { such that } \\
& \qquad \begin{aligned}
x & \left.+H S_{t+1}(\omega, \cdot) \geq \mathcal{E}_{t+1}(g)(\omega, \cdot) \mathbb{P}_{t}^{g}(\omega) \text {-a.s. }\right\}, \\
\pi^{\mathfrak{P}_{t}(\omega)}(g)=\inf \{x \in \mathbb{R}: & \exists H \in \mathbb{R}^{d} \text { such that } \\
& \left.x+H \Delta S_{t+1}(\omega, \cdot) \geq \mathcal{E}_{t+1}(g)(\omega, \cdot) \mathfrak{P}_{t}(\omega) \text {-q.s. }\right\} .
\end{aligned}
\end{aligned}
$$

Clearly, $\pi^{\mathbb{P}_{t}^{g}(\omega)}(g) \leq \pi_{\mathfrak{P}_{t}(\omega)}(g)$. Now assume towards a contradiction that the inequality is strict and set $\varepsilon:=\pi^{\mathfrak{P}_{t}(\omega)}(g)-\pi^{\mathbb{P}_{t}^{g}(\omega)}(g)>0$. Furthermore, note that for a sequence $\left(K_{n}\right)_{n \in \mathbb{N}}$ of compact sets such that $K_{n} \uparrow \mathbb{R}^{d}$, we have

$$
\begin{aligned}
& \pi^{\left.\mathbb{P}_{t}^{g}(\omega)\right|_{K_{n}}}(g):=\inf \left\{x \in \mathbb{R}: \exists H \in \mathbb{R}^{d} \text { such that } x+H \Delta S_{t+1} \geq \mathcal{E}_{t+1}(g)\right. \\
& \text { holds } \left.\mathbb{P}_{t}^{g}(\omega)\left(\cdot \mid \Delta S_{t+1}(\omega, \cdot) \in K_{n}\right) \text {-a.s. }\right\} \\
& \uparrow \pi^{\mathbb{P}_{t}^{g}(\omega)}(g) \quad \text { as } n \rightarrow \infty, \\
& \pi^{\left.\mathfrak{P}_{t}(\omega)\right|_{K_{n}}}(g):=\inf \left\{x \in \mathbb{R}: \exists H \in \mathbb{R}^{d} \text { such that } x+H \Delta S_{t+1} \geq \mathcal{E}_{t+1}(g)\right. \\
& \text { holds } \left.\left.\mathfrak{P}_{t}(\omega)\right|_{K_{n}} \text {-q.s. }\right\} \\
& \uparrow \pi^{\mathfrak{P}_{t}(\omega)}(g) \text { as } n \rightarrow \infty,
\end{aligned}
$$

where

$$
\left.\mathfrak{P}_{t}(\omega)\right|_{K_{n}}:=\left\{\mathbb{P}\left[\cdot \mid \Delta S_{t+1}(\omega, \cdot) \in K_{n}\right]: \mathbb{P} \in \mathfrak{P}_{t}(\omega), \mathbb{P}\left[\Delta S_{t+1}(\omega, \cdot) \in K_{n}\right]>0\right\} .
$$




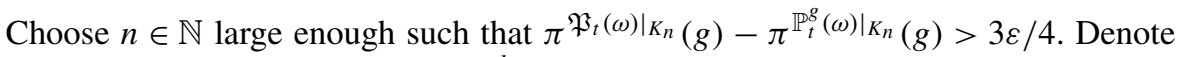
by $\mathcal{H}_{K_{n}}$ the closed set of all $H \in \mathbb{R}^{d}$ such that

$$
\pi^{\left.\mathbb{P}_{t}^{g}(\omega)\right|_{K_{n}}}(g)+\varepsilon / 2+H \Delta S_{t+1}(\omega, \cdot) \geq \mathcal{E}_{t+1}(g)(\omega, \cdot)
$$

$\mathbb{P}_{t}^{g}[\omega)\left(\cdot \mid \Delta S_{t+1}(\omega, \cdot) \in K_{n}\right]$-a.s. Then for every $H \in \mathcal{H}_{K_{n}}$ there exists $\mathbb{P}_{n}^{H} \in \mathfrak{P}_{t}(\omega)$ such that

$$
\begin{gathered}
\mathbb{P}_{n}^{H}\left[\left\{\pi^{\left.\mathbb{P}_{t}^{g}(\omega)\right|_{K n}}(g)+\varepsilon / 2+H \Delta S_{t+1}(\omega, \cdot)<\mathcal{E}_{t+1}(g)(\omega, \cdot)\right\}\right. \\
\left.\cap\left\{\Delta S_{t+1}(\omega, \cdot) \in K_{n}\right\}\right]>0 .
\end{gathered}
$$

Note that there exists a countable sequence $\left(H_{n}^{k}\right)_{k \in \mathbb{N}}$ which is dense in $\mathcal{H}_{K_{n}}$. In particular, for every $H \in \mathbb{R}^{d}$ such that we have $\mathbb{P}_{t}^{g}(\omega)\left[\cdot \mid \Delta S_{t+1}(\omega, \cdot) \in K_{n}\right]$-a.s.

$$
\pi^{\left.\mathbb{P}_{t}^{g}(\omega)\right|_{K_{n}}}(g)+\varepsilon / 4+H \Delta S_{t+1}(\omega, \cdot) \geq \mathcal{E}_{t+1}(g)(\omega, \cdot),
$$

there exists $k \in \mathbb{N}$ such that we have $\mathbb{P}_{t}^{g}(\omega)\left[\cdot \mid \Delta S_{t+1}(\omega, \cdot) \in K_{n}\right]$-a.s.

$$
\pi^{\left.\mathbb{P}_{t}^{g}(\omega)\right|_{K_{n}}}(g)+\varepsilon / 2+H_{n}^{k} \Delta S_{t+1}(\omega, \cdot) \geq \mathcal{E}_{t+1}(g)(\omega, \cdot) .
$$

Set now $\mathbb{P}^{n}=\sum_{k=1}^{\infty} 2^{-k} \mathbb{P}_{n}^{H_{n}^{k}} \in \mathcal{P}\left(X_{1}\right)$ and note that for all $n \in \mathbb{N}$ large enough,

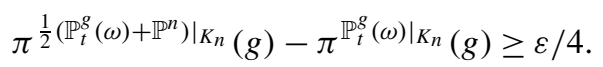

Taking $K_{n} \uparrow \mathbb{R}^{d}$, we have in particular

$$
\begin{aligned}
\mathcal{E}_{t}(g)(\omega) & \leq \sup \left\{\mathbb{E}_{\mathbb{Q}}[g]: \mathbb{Q} \approx \mathbb{P}_{t}^{g}(\omega), \mathbb{Q} \in \mathcal{M}_{X_{1}}\right\} \\
& <\lim _{n \rightarrow \infty} \sup \left\{\mathbb{E}_{\mathbb{Q}}[g]:\left.\mathbb{Q} \approx \frac{1}{2}\left(\mathbb{P}_{t}^{g}(\omega)+\mathbb{P}^{n}\right)\right|_{K_{n}}, \mathbb{Q} \in \mathcal{M}_{X_{1}}\right\} \\
& \leq \mathcal{E}_{t}(g)(\omega),
\end{aligned}
$$

which is a contradiction. Thus

$$
\mathcal{E}_{t}(g)(\omega)=\pi^{\mathfrak{P}_{t}(\omega)}(g)=\pi^{\mathbb{P}_{t}^{g}(\omega)}(g) .
$$

As $0 \in \operatorname{ri}\left(\operatorname{supp}\left(\mathbb{P}_{t}^{g}(\omega)\right)\right)$, a natural universally measurable candidate for a superhedging strategy $\omega \mapsto H_{t+1}(\omega)$ is the right derivative

$$
\lim _{\varepsilon \in \mathbb{Q}, \varepsilon \downarrow 0}\left(\mathcal{E}_{t}^{\varepsilon e_{i}}(g)(\omega)-\mathcal{E}_{t}(g)(\omega)\right) / \varepsilon,
$$

where $\mathcal{E}_{t}^{\varepsilon e_{i}}(g)(\omega)$ is the superhedging price for the Borel-measurable stock $\left(S_{t}+\varepsilon e_{i}\right)$, $i=1, \ldots, d^{\prime} \leq d$, instead of $S_{t}$. This is a pointwise limit of differences of upper semianalytic functions and thus universally measurable. For $\omega \in X_{t}$ such that this quantity does not exist, we set $H_{t+1}(\omega)=0$. Furthermore, in order to show that the 
map $\omega \mapsto \mathbb{P}_{t}^{g}(\omega)$ can be chosen to be universally measurable, we first note that in [12, Lemma 4.8], the set

$$
\begin{array}{r}
\left\{(\mathbb{Q}, \mathbb{P}) \in \mathcal{P}\left(\operatorname{proj}_{t+1}\left(\Sigma_{t}^{\omega}\right)\right) \times \mathcal{P}\left(\operatorname{proj}_{t+1}\left(\Sigma_{t}^{\omega}\right)\right): \mathbb{E}_{\mathbb{Q}}\left[\Delta S_{t+1}(\omega, \cdot)\right]=0,\right. \\
\left.\mathbb{P} \in \mathfrak{P}_{t}(\omega), \mathbb{Q} \ll \mathbb{P}\right\}
\end{array}
$$

is analytic. Thus we can apply the Jankov-von-Neumann selection theorem (see [8, Proposition 7.50]) to find $(1 / n)$-optimisers $\left(\mathbb{Q}_{t}^{n}(\omega), \mathbb{P}_{t}^{n}(\omega)\right)$ for $\mathcal{E}_{t}(g)(\omega)$ and the claim follows. The case $\Phi \neq 0$ can be handled by induction as in the proof of Theorem 2.5 for $\Phi \neq 0$.

In conclusion, we have found a strategy $(h, H) \in \mathcal{A}_{\Phi}\left(\mathbb{F}^{\mathcal{U}}\right)$ such that

$$
\sup _{\mathbb{Q} \in \mathcal{Q}_{\mathfrak{P}, \Phi}} \mathbb{E}_{\mathbb{Q}}[g]+h \cdot \Phi+\left(H \cdot S_{T}\right) \geq g \quad \text { P-q.s. }
$$

We now define

$$
\Omega_{g}^{\mathfrak{P}}=\Omega^{\mathfrak{P}} \cap\left\{\omega \in X: \sup _{\mathbb{Q} \in \mathcal{Q}_{\mathfrak{P}, \Phi}} \mathbb{E}_{\mathbb{Q}}[g]+h \cdot \Phi(\omega)+\left(H \cdot S_{T}\right)(\omega) \geq g(\omega)\right\} \in \mathcal{F}^{\mathcal{U}} .
$$

This concludes the proof.

Remark 4.13 By NA(P), Proposition 4.12 implies for $g=0$ that

$$
\begin{aligned}
& 0=\inf \left\{x \in \mathbb{R}: \exists(h, H) \in \mathcal{A}_{\Phi}\left(\mathbb{F}^{\mathcal{U}}\right)\right. \text { such that } \\
& \left.x+h \cdot \Phi+\left(H \cdot S_{T}\right) \geq 0 \mathfrak{P} \text {-q.s. }\right\} \\
& =\inf _{\tilde{g} \in \mathfrak{E}_{0}} \inf \left\{x \in \mathbb{R}: \exists(h, H) \in \mathcal{A}_{\Phi}\left(\mathbb{F}^{\mathcal{U}}\right)\right. \text { such that } \\
& \left.\qquad x+h \cdot \Phi+\left(H \bullet S_{T}\right) \geq \tilde{g} \text { on }\left(\Omega_{\tilde{g}}^{\mathfrak{P}}\right)_{\Phi}^{*}\right\} \\
& =\inf _{\tilde{g} \in \mathfrak{E}_{0}} \sup \left\{\mathbb{E}_{\mathbb{Q}}[\tilde{g}]: \mathbb{Q} \in \mathcal{M}_{\Omega_{\tilde{g}}^{\mathfrak{P}}, \Phi}\right\},
\end{aligned}
$$

where we define

$$
\mathfrak{E}_{0}=\left\{\tilde{g}: X \rightarrow(-\infty, 0] \mathcal{F}^{\mathcal{U}} \text {-measurable }: \tilde{g}=0 \mathfrak{P} \text {-q.s. }\right\} .
$$

In particular, for every $\tilde{g} \in \mathfrak{E}_{0}$, there exists $\mathbb{Q} \in \mathcal{M}_{X, \Phi}$ such that $\mathbb{E}_{\mathbb{Q}}[\tilde{g}]=0$. A similar result was obtained by Burzoni et al. [16] in a more general setup. Aggregating the martingale measures corresponding to all $\tilde{g}$ (and thus to all $\mathfrak{P}$-polar sets) to achieve a result comparable to Bouchard and Nutz [12] in a setup without using (APS) of $\mathfrak{P}$ remains an open problem.

Acknowledgements We thank Matteo Burzoni for his helpful remarks and comments. Johannes Wiesel further acknowledges support from the German Academic Scholarship Foundation. Part of this research was carried out while Jan Obłój was visiting the Sydney Mathematical Research Institute and he gratefully acknowledges their hospitality. 
Open Access This article is licensed under a Creative Commons Attribution 4.0 International License, which permits use, sharing, adaptation, distribution and reproduction in any medium or format, as long as you give appropriate credit to the original author(s) and the source, provide a link to the Creative Commons licence, and indicate if changes were made. The images or other third party material in this article are included in the article's Creative Commons licence, unless indicated otherwise in a credit line to the material. If material is not included in the article's Creative Commons licence and your intended use is not permitted by statutory regulation or exceeds the permitted use, you will need to obtain permission directly from the copyright holder. To view a copy of this licence, visit http://creativecommons.org/licenses/by/ $4.0 \%$.

\section{References}

1. Acciaio, B., Beiglböck, M., Penkner, F., Schachermayer, W.: A model-free version of the fundamental theorem of asset pricing and the super-replication theorem. Math. Finance 26, 233-251 (2016)

2. Bálint, D.Á., Schweizer, M.: Making no-arbitrage discounting-invariant: a new FTAP beyond NFLVR and NUPBR. Swiss Finance Institute Research Paper 18-23 (2018). Available online at https://doi.org/ 10.2139/ssrn.3676499

3. Bartl, D.: Exponential utility maximization under model uncertainty for unbounded endowments. Ann. Appl. Probab. 29, 577-612 (2019)

4. Bartl, D., Cheridito, P., Kupper, M., Tangpi, L.: Duality for increasing convex functionals with countably many marginal constraints. Banach J. Math. Anal. 11, 72-89 (2017)

5. Bayraktar, E., Zhang, Y.: Fundamental theorem of asset pricing under transaction costs and model uncertainty. Math. Oper. Res. 41, 1039-1054 (2016)

6. Bayraktar, E., Zhou, Z.: On arbitrage and duality under model uncertainty and portfolio constraints. Math. Finance 27, 988-1012 (2017)

7. Bayraktar, E., Zhang, Y., Zhou, Z.: A note on the fundamental theorem of asset pricing under model uncertainty. Risks 2, 425-433 (2014)

8. Bertsekas, D., Shreve, S.: Stochastic Optimal Control: The Discrete Time Case, vol. 23. Academic Press, New York (1978)

9. Black, F., Scholes, M.: The pricing of options and corporate liabilities. J. Polit. Econ. 81, 637-654 (1973)

10. Blanchard, R., Carassus, L.: No-arbitrage with multiple-priors in discrete time. Stoch. Process. Appl. 130, 6657-6688 (2020)

11. Bonnice, W., Reay, J.: Relative interiors of convex hulls. Proc. Am. Math. Soc. 20, 246-250 (1969)

12. Bouchard, B., Nutz, M.: Arbitrage and duality in nondominated discrete-time models. Ann. Appl. Probab. 25, 823-859 (2015)

13. Burzoni, M., Frittelli, M., Hou, Z., Maggis, M., Obłój, J.: Pointwise arbitrage pricing theory in discrete time. Math. Oper. Res. 43, 1034-1057 (2019)

14. Burzoni, M., Frittelli, M., Maggis, M.: Universal arbitrage aggregator in discrete-time markets under uncertainty. Finance Stoch. 20, 1-50 (2016)

15. Burzoni, M., Frittelli, M., Maggis, M.: Model-free superhedging duality. Ann. Appl. Probab. 27, 1452-1477 (2017)

16. Burzoni, M., Riedel, F., Soner, H.: Viability and arbitrage under Knightian uncertainty. Econometrica (2021). Forthcoming, available online at https://arxiv.org/abs/1707.03335

17. Carassus, L., Obłój, J., Wiesel, J.: The robust superreplication problem: a dynamic approach. SIAM J. Financ. Math. 10, 907-941 (2019)

18. Cassese, G.: Asset pricing with no exogenous probability measure. Math. Finance 18, 23-54 (2008)

19. Cont, R.: Model uncertainty and its impact on pricing derivative instruments. Math. Finance 16, 519-547 (2006)

20. Cox, A., Hou, Z., Oblój, J.: Robust pricing and hedging under trading restrictions and the emergence of local martingale models. Finance Stoch. 20, 669-704 (2016)

21. Cox, A., Obłój, J.: Robust pricing and hedging of double no-touch options. Finance Stoch. 15, 573-605 (2011)

22. Dalang, R.C., Morton, A., Willinger, W.: Equivalent martingale measures and no-arbitrage in stochastic securities market models. Stochastics 29, 185-201 (1990)

23. Davis, M., Hobson, D.: The range of traded option prices. Math. Finance 17, 1-14 (2007) 
24. Delbaen, F., Schachermayer, W.: A general version of the fundamental theorem of asset pricing. Math. Ann. 300, 463-520 (1994)

25. Denis, L., Martini, C.: A theoretical framework for the pricing of contingent claims in the presence of model uncertainty. Ann. Appl. Probab. 16, 827-852 (2006)

26. Dolinsky, Y., Soner, H.: Robust hedging with proportional transaction costs. Finance Stoch. 18, 327-347 (2014)

27. Föllmer, H., Schied, A.: Stochastic Finance: An Introduction in Discrete Time, 2nd edn. de Gruyter, Berlin (2011)

28. Hou, Z., Obłój, J.: Robust pricing-hedging dualities in continuous time. Finance Stoch. 22, 511-567 (2018)

29. Knight, F.: Risk, Uncertainty and Profit. Houghton Mifflin, Boston (1921)

30. Merton, R.C.: Rational theory of option pricing. Bell J. Econ. Manag. Sci. 4, 141-183 (1973)

31. Mykland, P.: Financial options and statistical prediction intervals. Ann. Stat. 31, 1413-1438 (2003)

32. Nutz, M.: Utility maximization under model uncertainty in discrete time. Math. Finance 26, 252-268 (2016)

33. Peng, S.: Nonlinear expectations, nonlinear evaluations and risk measures. In: Frittelli, M., Runggaldier, W. (eds.) Stochastic Methods in Finance. Lecture Notes in Mathematics, vol. 1856, pp. 165-253. Springer, Berlin (2004)

34. Riedel, F.: Financial economics without probabilistic prior assumptions. Decis. Econ. Finance 38, 75-91 (2015)

35. Rockafellar, R., Wets, R.: Variational Analysis, vol. 317. Springer, Berlin (2009)

36. Rokhlin, D.: A proof of the Dalang-Morton-Willinger theorem. Preprint (2008). Available online at https://arxiv.org/abs/0804.3308

37. Samuelson, P.: Rational theory of warrant pricing. Ind. Manage. Rev. 6(2), 13-39 (1965)

38. Shafer, G., Vovk, V.: Probability and Finance. It's Only a Game! John Wiley \& Sons, Hoboken (2005)

39. Smirnov, S.N.: A guaranteed deterministic approach to superhedging: a game equilibrium in the case of no trading constraints. J. Math. Sci. (N.Y.) 248, 105-115 (2020)

40. Vanderbei, R.: Uniform continuity is almost Lipschitz continuity. Technical report, SOR-91-11, Statistics and Operations Research Series (1997). Available online at https://vanderbei.princeton.edu/tex/ unif_cont/uc3.pdf

41. Vovk, V.: Continuous-time trading and the emergence of probability. Finance Stoch. 16, 561-609 (2012)

42. Wiesel, J.: Time-consistent data driven approach to robust pricing and hedging. DPhil thesis (2020). Available online at https://ora.ox.ac.uk/objects/uuid:b9df36d9-191c-4c61-84e7-9a69b6bbaf8a

Publisher's Note Springer Nature remains neutral with regard to jurisdictional claims in published maps and institutional affiliations. 